\title{
Geometric derivation of the microscopic stress: A covariant central force decomposition
}

\author{
Alejandro Torres-Sánchez ${ }^{\mathrm{a}}$, Juan M. Vanegas ${ }^{\mathrm{a}, 1}$, Marino Arroyo $^{\mathrm{a}}$ \\ ${ }^{a}$ LaCàN, Universitat Politècnica de Catalunya-BarcelonaTech, Barcelona, Spain
}

\begin{abstract}
We revisit the derivation of the microscopic stress, linking the statistical mechanics of particle systems and continuum mechanics. The starting point in our geometric derivation is the Doyle-Ericksen formula, which states that the Cauchy stress tensor is the derivative of the free-energy with respect to the ambient metric tensor and which follows from a covariance argument. Thus, our approach to define the microscopic stress tensor does not rely on the statement of balance of linear momentum as in the classical Irving-Kirkwood-Noll approach. Nevertheless, the resulting stress tensor satisfies balance of linear and angular momentum. Furthermore, our approach removes the ambiguity in the definition of the microscopic stress in the presence of multibody interactions by naturally suggesting a canonical and physically motivated force decomposition into pairwise terms, a key ingredient in this theory. As a result, our approach provides objective expressions to compute a microscopic stress for a system in equilibrium and for force-fields expanded into multibody interactions of arbitrarily high order. We illustrate the proposed
\end{abstract}

\footnotetext{
Email address: marino.arroyo@upc.edu (Marino Arroyo)

${ }^{1}$ Current Address: Center for Biological and Material Sciences, Sandia National Laboratories, Albuquerque, New Mexico
} 
methodology with molecular dynamics simulations of a fibrous protein using a force-field involving up to 5-body interactions.

Keywords: microscopic stress tensor, statistical mechanics, continuum mechanics, Doyle-Ericksen formula

\section{Introduction}

The increasing power of computers enables the atomistic simulation of material systems of growing size and complexity. However, it is difficult to interpret the physics of these systems from bare atomistic trajectories. In particular, there is a pressing need for coarse-grain measures of the effective
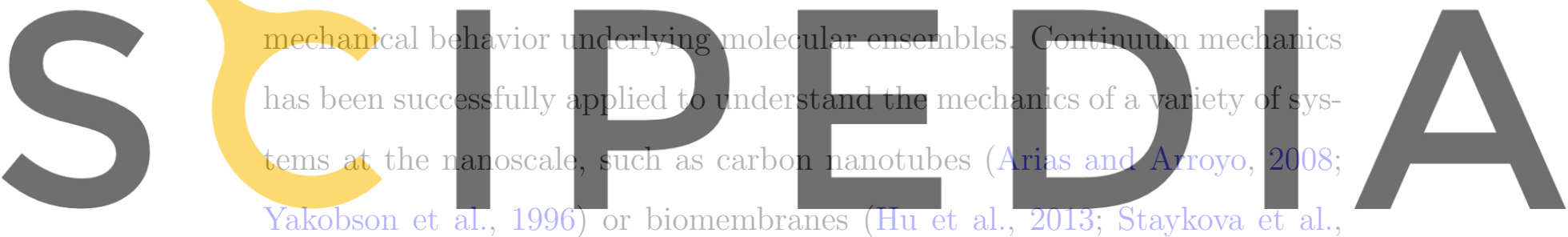

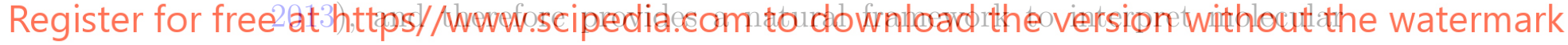

simulations of materials. In particular, local stress fields are routinely com-

puted from molecular simulations to understand the mechanics of different materials including defective crystals (Li et al., 2002; Pao et al., 2009; Song et al., 2013; Wei et al., 2012), lipid bilayers (Lindahl and Edholm, 2000; Vanegas et al., 2014), membrane proteins (Ollila et al., 2009; Vanegas and Arroyo, 2014), or isolated molecules (Edwards et al., 2012; Hatch and Debenedetti, 2012).

The mapping from the classical mechanics of a system of point-particles to a continuous stress field is usually understood in terms of the statistical mechanics framework pioneered by Irving and Kirkwood and further sub- 
stantiated by Noll, referred to here as the IKN procedure (Admal and Tadmor, 2010; Irving and Kirkwood, 1950; Noll, 1955; Schofield and Henderson, 1982). However, it is well-recognized that this mapping is not unique, particularly in the presence of complex force-fields (Admal and Tadmor, 2010; Schofield and Henderson, 1982). The major ambiguity in the theory comes from the non-unique decomposition of the interatomic forces from multibody potentials into pairwise terms. Different force decompositions have been proposed in the literature (Admal and Tadmor, 2010; Costescu and Gräter, 2013; Goetz and Lipowsky, 1998), which lead to stresses satisfying balance of linear momentum by construction. However, we have recently demonstrated
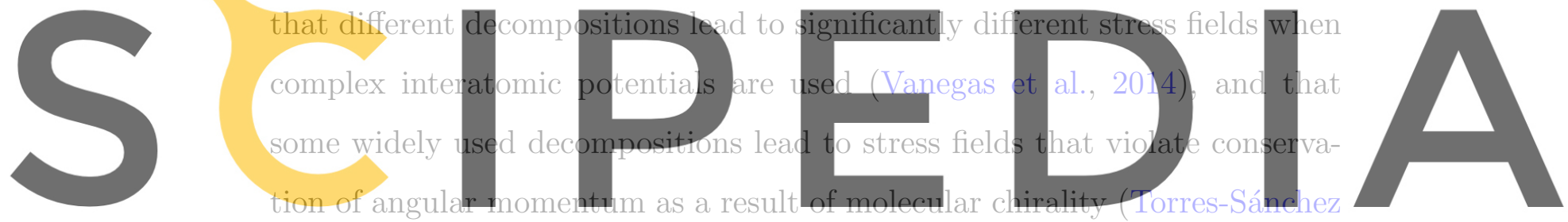

Register for free at https/)/wWw.scipedia.com fo download the version without the watermark force decomposition (CFD) (Admal and Tadmor, 2010; Tadmor and Miller, 2011), been proposed that provides symmetric stresses by construction, which therefore satisfy balance of angular momentum. The issue is not fully settled, however, because the CFD is not unique for potentials beyond 4-body interactions (Admal and Tadmor, 2010; Murdoch, 2012; Tadmor and Miller, 2011), which are nevertheless popular to model metals or proteins (Daw and Baskes, 1984; MacKerell et al., 2004).

Here, we propose an alternative geometric derivation of the microscopic Irving-Kirkwood stress, rooted in the Doyle-Ericksen relation of continuum mechanics (Doyle and Ericksen, 1956) rather than on the statement of bal- 
ance of linear momentum. See Baus and Lovett (1990, 1991); Mistura (1987) for related work. We further show that this approach allows us to canonically define the microscopic stress for arbitrary multibody potentials. Strikingly, our derivation does not resort to Noll's lemma, required in the IKN procedure, but leads to a specific instance of IKN stress corresponding to a distinguished central force decomposition that we call covariant central force decomposition (cCFD). This cCFD coincides with the common definition of the CFD for potentials with 4- or fewer-body interactions, fixes the gauge invariance of CFD for higher-order multibody potentials and provides physically meaningful stress fields (Torres-Sánchez et al., 2015).
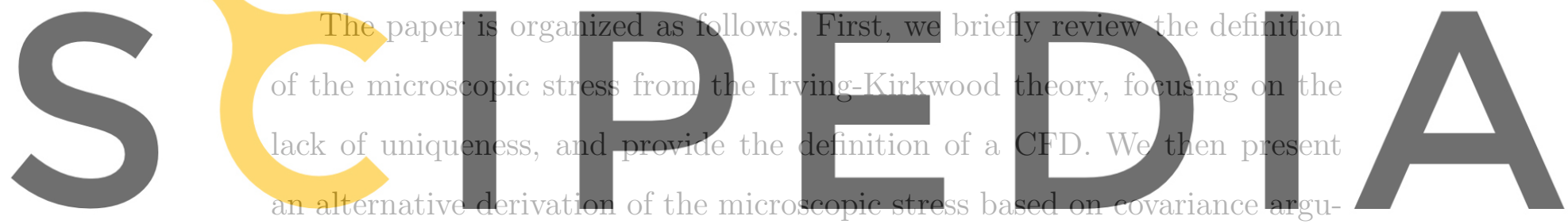

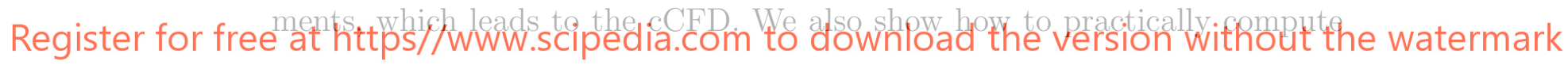

the cCFD for multibody potentials. Finally, we exercise the theory on a coiled-coil structural protein.

\section{Irving-Kirkwood theory: force decomposition and indefiniteness}

Let us briefly review the derivation of the stress tensor in the IrvingKirkwood theory. In this framework, the continuum density field is defined as

$$
\rho(\boldsymbol{x})=\sum_{\alpha=1}^{N}\left\langle m^{\alpha} \delta\left(\boldsymbol{r}^{\alpha}-\boldsymbol{x}\right)\right\rangle,
$$

where $\langle\cdot\rangle$ stands for an ensemble average over a non-equilibrium statisticalmechanics distribution, $m^{\alpha}$ and $\boldsymbol{r}^{\alpha}$ are the mass and position of particle $\alpha$, 
$\delta(\boldsymbol{x})$ is the 3D Dirac distribution centered at $\mathbf{0}$, and $N$ is the total number of particles in the system. Invoking the equivalence between the macroscopic momentum $\rho(x) \boldsymbol{v}(\boldsymbol{x})$ and the microscopic momentum $\sum_{\alpha}^{N}\left\langle m^{\alpha} \boldsymbol{v}^{\alpha} \delta\left(\boldsymbol{r}^{\alpha}-\boldsymbol{x}\right)\right\rangle$, the continuum velocity field is defined as

$$
\boldsymbol{v}(\boldsymbol{x})=\frac{1}{\rho(\boldsymbol{x})} \sum_{\alpha=1}^{N}\left\langle m^{\alpha} \boldsymbol{v}^{\alpha} \delta\left(\boldsymbol{r}^{\alpha}-\boldsymbol{x}\right)\right\rangle
$$

where $\boldsymbol{v}^{\alpha}$ is the velocity of particle $\alpha$. These two fields satisfy the continuity equation $\partial \rho / \partial t+\rho \nabla \cdot v=0$, expressing balance of mass in continuum mechanics, where $\nabla$. stands for the divergence operator. In the absence of external forces, the continuum balance of linear momentum requires that
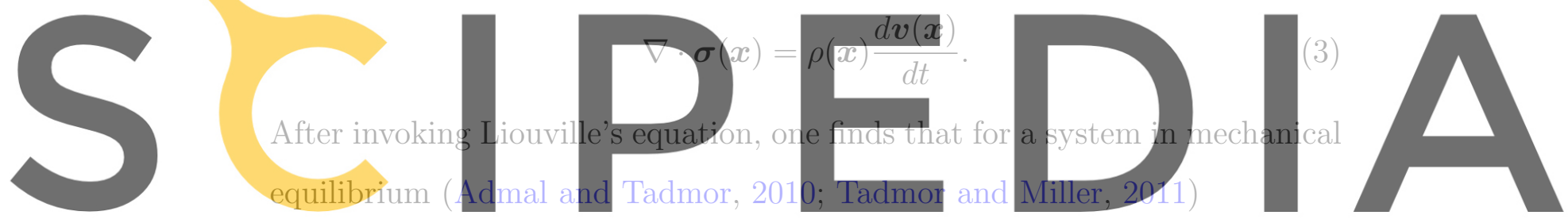

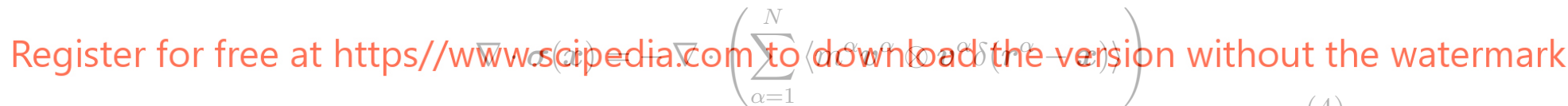

$$
+\sum_{\alpha=1}^{N}\left\langle\boldsymbol{F}^{\alpha} \delta\left(\boldsymbol{r}^{\alpha}-\boldsymbol{x}\right)\right\rangle
$$

where $\boldsymbol{F}^{\alpha}$ is the force on particle $\alpha$ and $\boldsymbol{a} \otimes \boldsymbol{b}$ denotes the dyadic product of vectors $\boldsymbol{a}$ and $\boldsymbol{b}$.

As discussed next, it is possible to obtain a statistical-mechanics expression of the right-hand side of Eq. (4) as the divergence of a tensor. Therefore, this expression provides a connection between the statistical mechanics of the particle system and the continuum stress tensor. However, this equation clearly provides a non-unique definition of $\boldsymbol{\sigma}$ since given any stress $\boldsymbol{\sigma}$ satisfying Eq. (4), we can add any divergence-free field $\boldsymbol{\omega}$, i.e. $\nabla \cdot \boldsymbol{\omega}=0$, to $\boldsymbol{\sigma}$ with 
the resulting field $\boldsymbol{\sigma}+\boldsymbol{\omega}$ also satisfying this equation. Therefore, with this approach we may only hope to rationally obtain an unambiguous definition of the stress tensor making as few arbitrary choices as possible.

An expression of $\boldsymbol{\sigma}$ that satisfies Eq. (4) by construction is (Irving and Kirkwood, 1950; Noll, 1955)

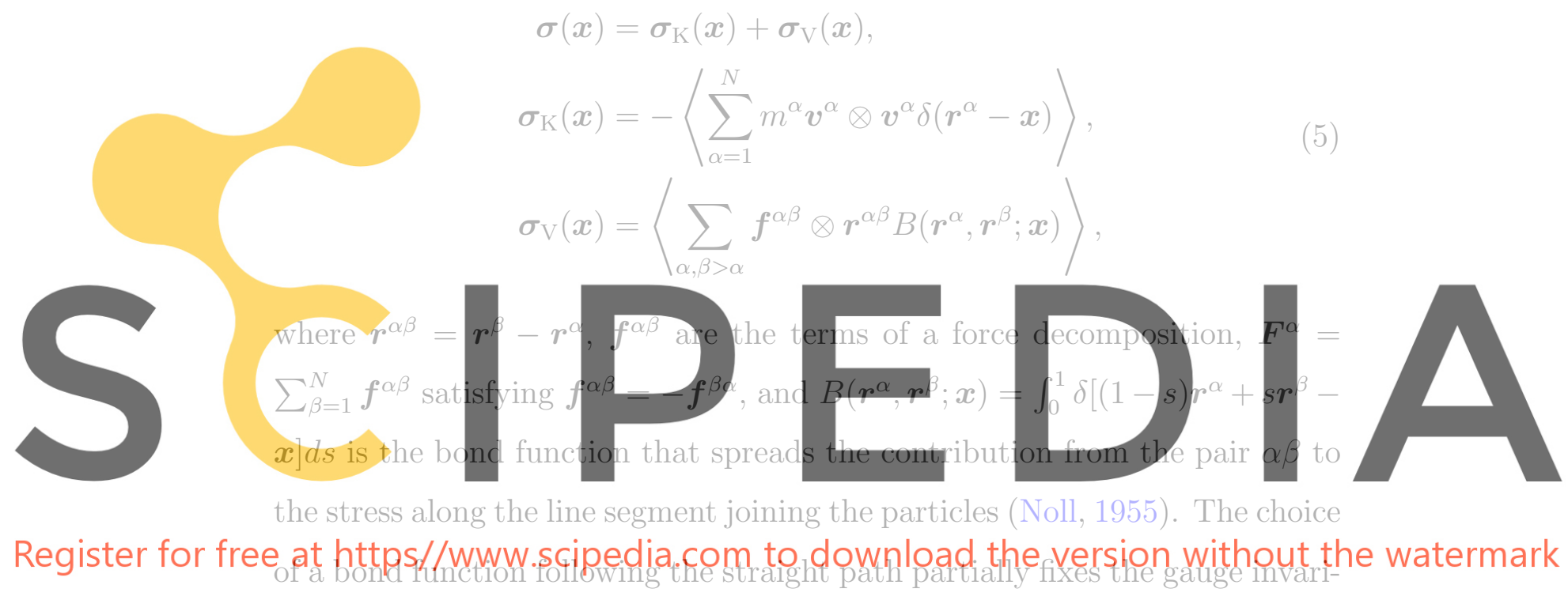

ance of $\sigma$. Recently, it has been shown that this is the only possible choice to obtain a stress field satisfying balance of angular momentum, i.e. $\sigma_{i j}=\sigma_{j i}$ (Admal and Tadmor, 2010). The gauge is completely fixed by choosing a force decomposition. Insisting on the symmetry of the stress field, this reference proposed the central force decomposition (CFD) as the only possible choice to obtain a symmetric stress by construction.

To define a CFD, let us express the potential energy of the system $V\left(\boldsymbol{r}^{1}, \ldots, \boldsymbol{r}^{N}\right)$ in terms of the set of distances defined by particles $\boldsymbol{r}^{1}, \ldots, \boldsymbol{r}^{N}$, which we denote by $\left\{r^{\alpha \beta}\right\}$ where $r^{\alpha \beta}=\left|\boldsymbol{r}^{\alpha \beta}\right|$. With such representation $\widetilde{V}\left(\left\{r^{\alpha \beta}\right\}\right)$, we 
can define the corresponding CFD as

$$
\boldsymbol{f}_{\mathrm{CFD}}^{\alpha \beta}=\varphi_{\alpha \beta} \hat{\boldsymbol{r}}^{\alpha \beta}
$$

where

$$
\varphi_{\alpha \beta}=\frac{\partial \widetilde{V}\left(\left\{r^{\alpha \beta}\right\}\right)}{\partial r^{\alpha \beta}}
$$

and $\hat{\boldsymbol{r}}^{\alpha \beta}=\boldsymbol{r}^{\alpha \beta} / r^{\alpha \beta}$. The representation of a potential in terms of distances always exists as result of the invariance with respect to rigid body transformations of classical potentials (Tadmor and Miller, 2011). Note that, since $f^{\alpha \beta}$ is parallel to $\boldsymbol{r}^{\alpha \beta}$, the symmetry of the stress is apparent recalling Eq. (5).

However, this CFD has an important limitation for systems with $N>4$.
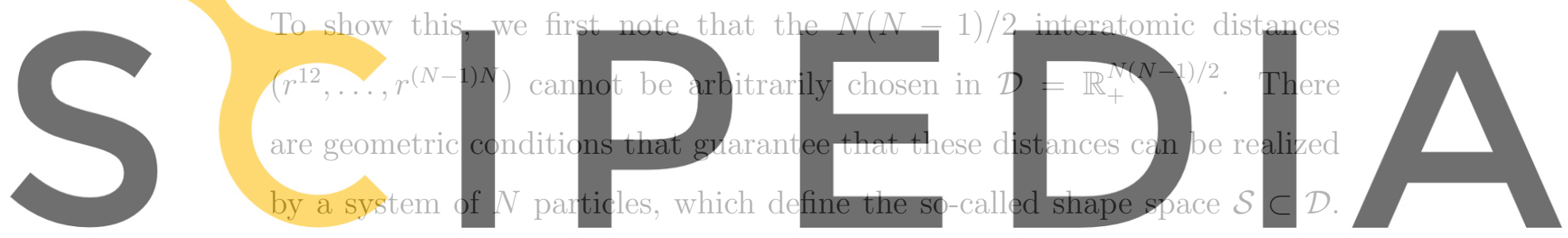

When $N>4$, the dimension of the manifold $\mathcal{S}$ is smaller than $N(N-1) / 2$.

Register for free at https//www.scipedia.com to download the version without the watermark

and therelore the differential calculus involved in Eq. (7) needs to be care-

fully considered (Littlejohn and Reinsch, 1997; Porta et al., 2005). Noting

this fact, it has been argued that, to be able to take the partial derivative $\partial \widetilde{V}\left(\left\{r^{\alpha \beta}\right\}\right) / \partial r^{\alpha \beta}$ in Eq. (6), the potential needs to be extended to $\mathcal{D}$ (Admal and Tadmor, 2010; Tadmor and Miller, 2011). However, when $N>4$, there exists infinitely many extensions, leading to an extension-dependent force decomposition and an ambiguous definition of the stress, all of which differ by divergence-free fields. The lack of a rational procedure to fix this gauge freedom has been a source of criticism (Murdoch, 2012).

A natural way to alleviate the massive non-uniqueness associated with potential extensions is to perform the CFD independently on each of the terms 
of a multibody expansion of the potential energy. Suppose for instance that $V$ is expressed in terms of contributions involving up to four particles, as it is often the case in classical models of biomolecules. In this case, even though the shape space $\mathcal{S}$ involving all particles in the system is a complex manifold, each potential contribution only sees $n$ particles $(n \leq 4)$, which define a trivial local shape space. Thus, each of these terms can be uniquely expressed in terms of interatomic distances and the CFD is unique for this potential contribution (Vanegas et al., 2014). An alternative potential extension of $V$ will in general couple particles not interacting through the terms of the original multibody expansion.
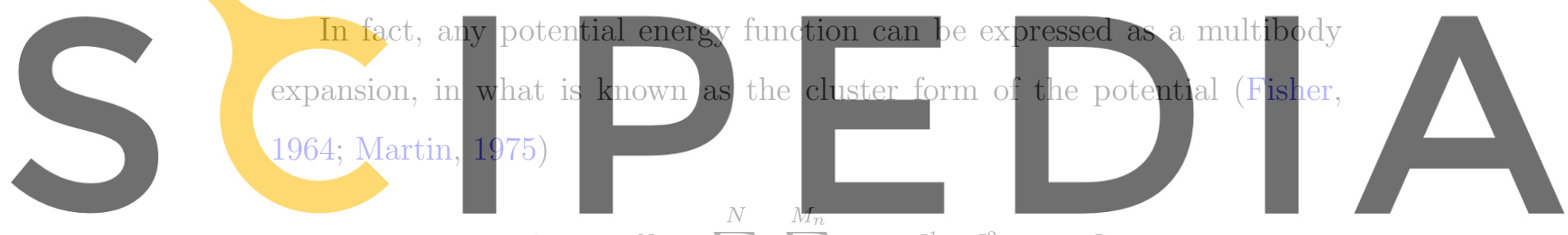

Register for free at https//www.scipedia.com to $\Phi_{n}$ download the version without the watermark

where each $V_{I_{n}}$ is a $n$-body potential, $M_{n} \leq\left(\begin{array}{l}N \\ n\end{array}\right)$ is the number $n$-body interactions amongst the $N$ particles, and $I_{n}^{1}, I_{n}^{2}, \ldots, I_{n}^{n}$ label the $n$ particles interacting through $V_{I_{n}}$. This cluster expansion can be made unique by requiring that the potentials $V_{I_{n}}\left(\boldsymbol{r}^{I_{n}^{1}}, \boldsymbol{r}^{I_{n}^{2}}, \ldots, \boldsymbol{r}^{I_{n}^{n}}\right)$ vanish whenever any of the intervening particles is brought infinitely far away from the rest. Is this were not true, then such a potential would not be a genuine $n$-body potential, but rather $n-1$ or lower (Martin, 1975). With this definition, $V_{I_{2}}$ represents the interaction of two isolated atoms, $V_{I_{3}}$ is the excess of energy of an isolated triplet of atoms not accounted by their pair interactions, and in 
general $V_{I_{n}}$ represents the part of the interaction energy of $n$ particles minus the interaction energy from all their $n-1, n-2, \ldots, 2$-body interactions. Within this formalism, the CFD can be written as

$$
\boldsymbol{f}_{\mathrm{CFD}}^{\alpha \beta}=\sum_{n=2}^{N} \sum_{I_{n}=1}^{M_{n}} \varphi_{\alpha \beta}^{I_{n}} \hat{\boldsymbol{r}}^{\alpha \beta}
$$

where $\widetilde{V}_{I_{n}}\left(\left\{r^{\alpha \beta}\right\}\right)$ is a representation of the interatomic potential $V_{I_{n}}\left(\left\{r^{\alpha}\right\}\right)$ and

$$
\varphi_{\alpha \beta}^{I_{n}}=\frac{\partial \widetilde{V}_{I_{n}}\left(\left\{r^{\alpha \beta}\right\}\right)}{\partial r^{\alpha \beta}} .
$$

We note that if the cluster expansion has non-zero terms with $n>4$, this
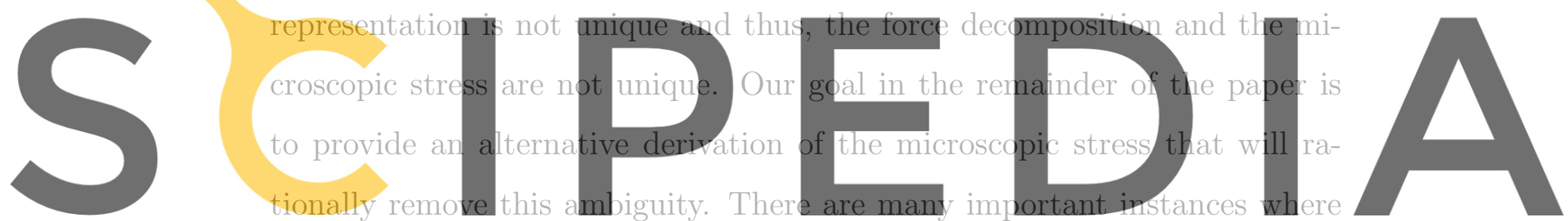

$n>4$. For example, classical models of biomolecules include 5-body interRegister for free at https//www.scipedia.com to download the version without the watermark actions to improve structural accuracy compared to experimental measure-

ments (MacKerell et al., 2004). For force-fields based on functional-density or semi-empirical formalisms, such as the embedded atom method (Daw and Baskes, 1984), the cluster form is not explicit, but can be computed systematically (Fisher, 1964; Martin, 1975) and will involve higher-order terms.

\section{A geometric derivation of the microscopic stress}

\subsection{The microscopic stress from the Doyle-Ericksen formula}

We consider a system at equilibrium in a NVT ensemble enclosed by three-dimensional volume $\Omega$. For simplicity, we consider a periodic system 
and follow the minimum image convention, i.e. particle $\alpha$ interacts with the closest image of particle $\beta$.

We define the microscopic stress as the statistical mechanics equivalent of the so-called Doyle-Ericksen formula in continuum mechanics (Doyle and Ericksen, 1956; Marsden and Hughes, 1983; Yavari and Marsden, 2012; Yavari et al., 2006), in an approach similar in spirit to previous variational theories (Baus and Lovett, 1990, 1991; Mistura, 1987). The Doyle-Ericksen formula expresses the Cauchy stress tensor as

$$
\sigma^{i j}=2 \frac{\partial a}{\partial g_{i j}},
$$
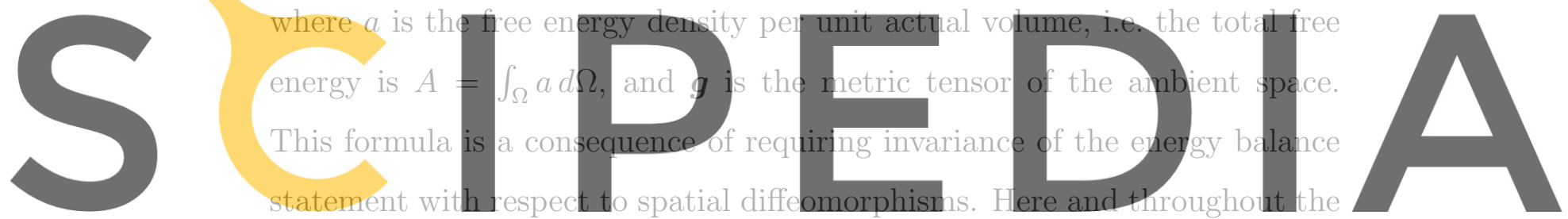

Register paper lower and upper indices refer to covariant and contravariant compoe at https//www.scipedia.com to download the version without the watermark nents, and $g^{i j}$ denotes the components of the inverse of the metric tensor,

satisfying $g^{i j} g_{j k}=\delta_{k}^{i}$.

For our molecular system, the canonical free energy takes the form

$$
A=-k_{B} T \log Z
$$

where $k_{B}$ is Boltzmann's constant, $T$ is the absolute temperature and $Z$ is the partition function

$$
Z=\int e^{-H(\boldsymbol{r}, \boldsymbol{p}) /\left(k_{B} T\right)} d \boldsymbol{r} d \boldsymbol{p}
$$

In this equation $\boldsymbol{r}=\left(\boldsymbol{r}^{1}, \ldots, \boldsymbol{r}^{N}\right)$ are the particle positions, $\boldsymbol{p}=\left(\boldsymbol{p}^{1}, \ldots, \boldsymbol{p}^{N}\right)$ are the momenta, and $H=K+V$ is the Hamiltonian, which we assume to 
be separable. For such a particle system, the notion of free energy density involved in the Doyle-Ericksen formula in Eq. (11) is unclear. Nevertheless, it is straightforward to modify the derivation of the Doyle-Ericksen formula in the continuum case to obtain a more general form not requiring the existence of such a density in terms of a functional derivative as

$$
\sigma^{i j}=2 \frac{\delta A}{\delta g_{i j}} .
$$

As we show next, this equation is pertinent to molecular systems because $A$ is indeed a functional of $\boldsymbol{g}$.

Being a fundamental covariance requirement of the theory of continuum mechanics, Eq. (14) is a legitimate starting point to define the microscopic stress, alternative to the continuum statement of linear momentum invoked by the more standard IKN approach, c.f. Eq. (4). To exercise this idea, we need to provide a statistical mechanics evaluation of the right-hand side of Eq. (14). For this, one may consider from the outset a particle system defined on a general Riemannian manifold, in which $H$ and $A$ will necessarily depend on the metric tensor (Yavari and Marsden, 2009). Here, however, we focus on particle systems evolving in Euclidean space. In this case, the dependence on $\boldsymbol{g}$ emerges when $A$ is expressed covariantly, i.e. for an arbitrary coordinate system, which then allows us to take the functional derivative.

\subsection{The free energy in a general coordinate system}

We analyze next the dependence of the free energy on the ambient metric. For that, we follow the passive approach of Doyle and Ericksen (1956) and introduce an arbitrary change of variables in space, characterized by the diffeomorphism $\xi(\boldsymbol{x})$ from $\Omega$ onto itself. This change of variables induces a 
canonical point transformation in phase space

$$
\begin{array}{r}
\hat{\boldsymbol{r}}^{\alpha}=\xi\left(\boldsymbol{r}^{\alpha}\right), \\
\hat{\boldsymbol{p}}^{\alpha}=\left(D \xi\left(\boldsymbol{r}^{\alpha}\right)\right)^{-T} \boldsymbol{p}^{\alpha} \quad \text { or } \quad p_{i}^{\alpha}=D_{i} \xi^{j}\left(\boldsymbol{r}^{\alpha}\right) \hat{p}_{j}^{\alpha},
\end{array}
$$

for each particle. Note that momenta transform like co-vectors or one-forms. It immediately follows that $d \hat{\boldsymbol{r}}=[\operatorname{det}(D \xi)]^{N} d \boldsymbol{r}$ and $d \hat{\boldsymbol{p}}=[\operatorname{det}(D \xi)]^{-N} d \boldsymbol{p}$, and therefore this transformation leaves the phase volume element unchanged

$$
d \hat{\boldsymbol{r}} d \hat{\boldsymbol{p}}=d \boldsymbol{r} d \boldsymbol{p}
$$

We consider a standard form for the kinetic energy in the initial coordinate system

$$
K\left(\boldsymbol{r}, \boldsymbol{p} ; \boldsymbol{g}_{0}\right)=\sum_{\alpha=1}^{N} \frac{1}{2 m^{\alpha}} g_{0}^{i j}\left(\boldsymbol{r}^{\alpha}\right) p_{i}^{\alpha} p_{j}^{\alpha},
$$

where $\boldsymbol{g}_{0}$ the metric tensor associated to the initial coordinates $\left\{x^{i}\right\}$. In practice, this coordinate system is Cartesian and $g_{0}^{i j}=\delta^{i j}$. Inserting Eq. (16) into Eq. (18), we obtain

$$
\begin{aligned}
K\left(\boldsymbol{r}, \boldsymbol{p} ; \boldsymbol{g}_{0}\right) & =\sum_{\alpha=1}^{N} \frac{1}{2 m^{\alpha}} g_{0}^{i j}\left(\xi^{-1}\left(\hat{\boldsymbol{r}}^{\alpha}\right)\right) D_{i} \xi^{k} D_{j} \xi^{l} \hat{p}_{k}^{\alpha} \hat{p}_{l}^{\alpha}, \\
& =\sum_{\alpha=1}^{N} \frac{1}{2 m^{\alpha}} g^{k l}\left(\hat{\boldsymbol{r}}^{\alpha}\right) \hat{p}_{k}^{\alpha} \hat{p}_{l}^{\alpha}=K(\hat{\boldsymbol{r}}, \hat{\boldsymbol{p}} ; \boldsymbol{g})
\end{aligned}
$$

where we have defined $\boldsymbol{g}$ as the push-forward by the mapping $\xi$ of the original metric tensor

$$
\boldsymbol{g}=\xi_{*} \boldsymbol{g}_{0}=D \xi^{-T}\left(\boldsymbol{g}_{0} \circ \xi^{-1}\right) D \xi^{-1}
$$

It is easy to see that $\xi$ is an isometry between $\left(\Omega, \boldsymbol{g}_{0}\right)$ and $(\Omega, \boldsymbol{g})$, and therefore if $\left(g_{0}\right)_{i j}=\delta_{i j}$ is the standard Euclidean metric, then $\boldsymbol{g}=D \xi^{-T} D \xi^{-1}$ is 
the expression of the Euclidean metric in the coordinates given by $\xi$. In conclusion, the kinetic energy takes the same form in the original and in the new variables, provided the appropriate metric tensor is considered.

As for the potential energy, we consider for definiteness the cluster form of the potential in Eq. (8) and express each cluster potential in terms of particle distances

$$
V=\sum_{n=2}^{N} \sum_{I_{n}=1}^{M_{n}} \widetilde{V}_{I_{n}}\left(\left\{r^{\alpha \beta}\right\}\right)
$$

where $\widetilde{V}_{I_{n}}$ is any extension of the potential contribution $V_{I_{n}}$. To express $V$ covariantly, we note that irrespective of the coordinate system, i.e. for any metric tensor given as in Eq. (20), $r^{\alpha \beta}$ is the length of the geodesic curve joining points $\hat{\boldsymbol{r}}^{\alpha}$ and $\hat{\boldsymbol{r}}^{\beta}$, which we denote by $\boldsymbol{c}_{\boldsymbol{g}}(\lambda)$ for $\lambda \in[0,1]$, emphasizing its dependence on $\boldsymbol{g}$ (Do Carmo, 1992). Since here $\boldsymbol{g}$ is the expression of the standard Euclidean metric in a general coordinate system, there exists a single geodesic joining any two particles (the straight line of Euclidean space described in the general coordinate system). Thus, the distance between two particles can be written as

$$
r^{\alpha \beta}\left(\hat{\boldsymbol{r}}^{\alpha}, \hat{\boldsymbol{r}}^{\beta} ; \boldsymbol{g}\right)=\int_{0}^{1} \sqrt{\left[g_{s}\right]_{i j}\left(\boldsymbol{c}_{\boldsymbol{g}}(\lambda)\right) \partial_{\lambda} c_{\boldsymbol{g}}^{i}(\lambda) \partial_{\lambda} c_{\boldsymbol{g}}^{j}(\lambda)} d \lambda,
$$

which clearly shows that $r^{\alpha \beta}$, and hence $V, H$, and $A$, are functionals of $\boldsymbol{g}$. Because $\xi$ is an isometry between $\left(\Omega, \boldsymbol{g}_{0}\right)$ and $(\Omega, \boldsymbol{g})$,

$$
r^{\alpha \beta}\left(\hat{\boldsymbol{r}}^{\alpha}, \hat{\boldsymbol{r}}^{\beta} ; \boldsymbol{g}\right)=r^{\alpha \beta}\left(\boldsymbol{r}^{\alpha}, \boldsymbol{r}^{\beta} ; \boldsymbol{g}_{0}\right)
$$

and $\boldsymbol{c}_{\boldsymbol{g}}=\xi \circ \boldsymbol{c}_{\boldsymbol{g}_{0}}$.

Thus, if we define

$$
H\left(\boldsymbol{r}, \boldsymbol{p} ; \boldsymbol{g}_{0}\right)=K\left(\boldsymbol{r}, \boldsymbol{p} ; \boldsymbol{g}_{0}\right)+\sum_{n=2}^{N} \sum_{I_{n}=1}^{M_{n}} \widetilde{V}_{I_{n}}\left(\left\{r^{\alpha \beta}\left(\boldsymbol{r}^{\alpha}, \boldsymbol{r}^{\beta} ; \boldsymbol{g}_{0}\right)\right\}\right)
$$


and

$$
H(\hat{\boldsymbol{r}}, \hat{\boldsymbol{p}} ; \boldsymbol{g})=K(\hat{\boldsymbol{r}}, \hat{\boldsymbol{p}} ; \boldsymbol{g})+\sum_{n=2}^{N} \sum_{I_{n}=1}^{M_{n}} \widetilde{V}_{I_{n}}\left(\left\{r^{\alpha \beta}\left(\hat{\boldsymbol{r}}^{\alpha}, \hat{\boldsymbol{r}}^{\beta} ; \boldsymbol{g}\right)\right\}\right),
$$

we have shown that $H\left(\boldsymbol{r}, \boldsymbol{p} ; \boldsymbol{g}_{0}\right)=H(\hat{\boldsymbol{r}}, \hat{\boldsymbol{p}} ; \boldsymbol{g})$. Performing the change of variables given by Eqs. $(15,16)$, this fact and Eq. (17) allow us to express the canonical free energy of the system as

$$
\begin{aligned}
A & =-k_{B} T \log \int e^{-H\left(\boldsymbol{r}, \boldsymbol{p} ; \boldsymbol{g}_{\mathbf{0}}\right) /\left(k_{B} T\right)} d \boldsymbol{r} d \boldsymbol{p} \\
& =-k_{B} T \log \int e^{-H(\hat{\boldsymbol{r}}, \hat{\boldsymbol{p}} ; \boldsymbol{g}) /\left(k_{B} T\right)} d \hat{\boldsymbol{r}} d \hat{\boldsymbol{p}} \\
& =-k_{B} T \log \int e^{-H(\boldsymbol{r}, \boldsymbol{p} ; \boldsymbol{g}) /\left(k_{B} T\right)} d \boldsymbol{r} d \boldsymbol{p}
\end{aligned}
$$

where in the last step we have just changed the notation for the integration variables. Examining the first and last lines, we observe that although the free energy is a functional of the metric tensor $A[\boldsymbol{g}]$, it is independent of it as long as it is induced by a change of coordinates as in Eq. (20). We can also see that the diffeomorphism $\xi$ in this theory is just a tool to generate an admissible change of the ambient metric tensor, without moving or changing the coordinates of the particles themselves. We examine next the physical consequences of these facts.

\subsection{Statistical mechanics representation of the microscopic stress and me- chanical equilibrium}

To compute the functional derivative in Eq. (14) we consider a family of changes of coordinates $\xi_{s}(\boldsymbol{x})$ from $\Omega$ onto itself, parametrized by $s$, and such that at $s=0, \xi_{0}^{i}(\boldsymbol{x})=x^{i}$ is the identity map. The mappings $\xi_{s}$ generate by push-forward admissible changes of the ambient metric tensor $\boldsymbol{g}_{s}$ as in Eq. (20), making the free energy $A$ effectively a function of $s$ alone, see Eq. (26). 
Denoting by $\eta^{i}(\boldsymbol{x})=\left.\partial_{s} \xi_{s}^{i}(\boldsymbol{x})\right|_{s=0}$ the rate of change of the coordinate system at the identity, it follows that (Do Carmo, 1992; Marsden and Hughes, 1983)

$$
\left.\frac{d}{d s}\right|_{s=0}\left(g_{s}\right)_{i j}=\left[\mathcal{L}_{\eta}\left(\boldsymbol{g}_{0}\right)\right]_{i j}=\frac{1}{2}\left[\nabla_{i} \eta_{j}+\nabla_{j} \eta_{i}\right],
$$

where $\mathcal{L}_{\boldsymbol{\eta}}(\boldsymbol{g})$ is the Lie derivative of the metric along the vector field $\boldsymbol{\eta}$. Thus, the metric variation is characterized by $\boldsymbol{\eta}$ and the functional derivative of any functional depending on the metric tensor, $F[\boldsymbol{g}]$, is given by the relation

$$
\left.\frac{d}{d s}\right|_{s=0} F\left[\boldsymbol{g}_{s}\right]=\int_{\Omega} \frac{\delta F}{\delta g_{i j}}\left[\mathcal{L}_{\boldsymbol{\eta}}\left(\boldsymbol{g}_{0}\right)\right]_{i j} d \Omega=\int_{\Omega} \frac{\delta F}{\delta g_{i j}} h_{i j} d \Omega,
$$

where we have introduced the shorthand notation $\boldsymbol{h}=\mathcal{L}_{\eta}\left(\boldsymbol{g}_{0}\right)$.

Recalling Doyle-Ericksen formula in Eq. (14), the form of the canonical free energy in Eq. (12), and Eq. (28), we have

$$
\begin{aligned}
\int_{\Omega} \frac{1}{2} \boldsymbol{\sigma}: \boldsymbol{h} d \Omega & =\int_{\Omega} \frac{\delta A}{\delta \boldsymbol{g}}: \boldsymbol{h} d \Omega=\left.\frac{d}{d s}\right|_{s=0} A\left[\boldsymbol{g}_{s}\right] \\
& =-\frac{k_{B} T}{Z} \int-\frac{1}{k_{B} T}\left(\left.\frac{d}{d s}\right|_{s=0} H\left(\boldsymbol{r}, \boldsymbol{p} ; \boldsymbol{g}_{s}\right)\right) e^{-H\left(\boldsymbol{r}, \boldsymbol{p} ; \boldsymbol{g}_{0}\right) /\left(k_{B} T\right)} d \boldsymbol{r} d \boldsymbol{p} \\
& =\left\langle\left.\frac{d}{d s}\right|_{s=0} H\left(\boldsymbol{r}, \boldsymbol{p} ; \boldsymbol{g}_{s}\right)\right\rangle=\left\langle\int_{\Omega} \frac{\delta H}{\delta \boldsymbol{g}}: \boldsymbol{h} d \Omega\right\rangle=\int_{\Omega}\left\langle\frac{\delta H}{\delta \boldsymbol{g}}\right\rangle: \boldsymbol{h} d \Omega
\end{aligned}
$$

Thus, by defining the instantaneous microscopic stress tensor as

$$
\boldsymbol{\sigma}_{\text {inst }}=2 \frac{\delta H}{\delta \boldsymbol{g}}=\underbrace{2 \frac{\delta K}{\delta \boldsymbol{g}}}_{\boldsymbol{\sigma}_{K, \text { inst }}}+\underbrace{2 \frac{\delta V}{\delta \boldsymbol{g}}}_{\boldsymbol{\sigma}_{V, \text { inst }}},
$$

we can represent the microscopic stress tensor as $\boldsymbol{\sigma}=\boldsymbol{\sigma}_{K}+\boldsymbol{\sigma}_{V}$, where the kinetic and potential contributions are the ensemble averages $\boldsymbol{\sigma}_{K}=\left\langle\boldsymbol{\sigma}_{K, \text { inst }}\right\rangle$ and $\boldsymbol{\sigma}_{V}=\left\langle\boldsymbol{\sigma}_{V, \text { inst }}\right\rangle$.

This definition of the stress tensor obviously satisfies balance of angular momentum because it is symmetric by construction. Furthermore, comparing 
Eqs. $(25,26)$ and recalling Eq. (27), it is clear that

$$
0=\left.\frac{d}{d s}\right|_{s=0} A\left[\boldsymbol{g}_{s}\right]=\int_{\Omega} \frac{1}{2} \boldsymbol{\sigma}: \nabla \boldsymbol{\eta} d \Omega,
$$

which should hold for any infinitesimal change of coordinates $\boldsymbol{\eta}$. This is the weak form of balance of linear momentum for the microscopic stress.

\subsection{Uniqueness of the variational definition of the stress tensor}

We emphasize that here we consider a system evolving in standard Euclidean space but described by a general set of curvilinear coordinates. For this reason, when computing the functional derivative of $A$ we only consider metric changes characterized by Eq. (27), which are not the most general metric variations (i.e. merely symmetric tensor fields). As we discuss next, this fact is related to the fundamental non-uniqueness of the microscopic stress tensor, also present in the proposed formalism despite previous claims (Mistura, 1987; Rossi and Testa, 2010). Indeed, let $\mathcal{L}_{2}$ be the completion of the Hilbert space of symmetric tensors with the scalar product

$$
(\boldsymbol{\alpha}, \boldsymbol{\beta})=\int_{\Omega} \alpha^{i j} \beta_{i j} d \Omega .
$$

This space admits the orthogonal decomposition $\mathcal{L}_{2}=\mathcal{L}_{2}^{\|} \oplus \mathcal{L}_{2}^{\perp}$, where

$$
\mathcal{L}_{2}^{\|}=\left\{\boldsymbol{h} \mid h_{i j}=\frac{1}{2}\left[\nabla_{i} \eta_{j}+\nabla_{j} \eta_{i}\right]\right\},
$$

are the metric variations induced by diffeomorphisms and

$$
\mathcal{L}_{2}^{\perp}=\{\boldsymbol{\omega} \mid \nabla \cdot \boldsymbol{\omega}=\mathbf{0}\}
$$

are perpendicular to them (Berger and Ebin, 1969). As a direct consequence, the functional derivative $\delta A / \delta \boldsymbol{g}$ in Eq. (29) cannot be uniquely identified 
since we can add to it any field in $\mathcal{L}_{2}^{\perp}$ without altering the variation of the A. Physically, adding a self-equilibrated stress field does not perform work against an infinitesimal metric variation in $\mathcal{L}_{2}^{\|}$.

Considering general variations of the metric would remove this indeterminacy, but such variations would bring the system out of the shape space, where interatomic potentials are not intrinsically defined. In other words, metric variations not induced by changes of coordinates would result in pairwise distances that cannot be embedded in Euclidean space, and thus it would not make physical sense to evaluate $\widetilde{V}\left(\left\{r^{\alpha \beta}\right\}\right)$ at these pairwise distances. Despite this fundamental indeterminacy also present in the IKN procedure, we show next that the method presented here provides a rational and unambiguous definition of the stress, and leads to a unique central force decomposition irrespective of the number of particles intervening in the potential.

We also see from this discussion that, since $\boldsymbol{\sigma}$ satisfies Eq. (31), $\boldsymbol{\sigma} \in \mathcal{L}_{2}^{\perp}$. In other words, $\boldsymbol{\sigma}$ satisfies the strong form of the balance of linear momentum $\operatorname{div} \boldsymbol{\sigma}=0$.

\subsection{Kinetic part of the microscopic stress}

We focus now on the kinetic contribution to the stress tensor

$$
\boldsymbol{\sigma}_{K}=2\left\langle\frac{\delta K}{\delta \boldsymbol{g}}\right\rangle
$$

As previously discussed,

$$
K\left(\boldsymbol{r}, \boldsymbol{p} ; \boldsymbol{g}_{s}\right)=\sum_{\alpha=1}^{N} \frac{1}{2 m^{\alpha}} g_{s}^{i j}\left(\boldsymbol{r}^{\alpha}\right) p_{i}^{\alpha} p_{j}^{\alpha}
$$


which can be formally expressed as a functional depending on $\boldsymbol{g}(\boldsymbol{x})$ using Dirac distributions $\delta\left(\boldsymbol{r}^{\alpha}-\boldsymbol{x}\right)$,

$$
K\left(\boldsymbol{r}, \boldsymbol{p} ; \boldsymbol{g}_{s}\right)=\sum_{\alpha=1}^{N} \frac{1}{2 m^{\alpha}} \int_{\Omega} g_{s}^{i j}(\boldsymbol{x}) p_{i}^{\alpha} p_{j}^{\alpha} \delta\left(\boldsymbol{x}-\boldsymbol{r}^{\alpha}\right) d \Omega .
$$

The variation produced by a change of metric $\boldsymbol{h}=\mathcal{L}_{\boldsymbol{\eta}}(\boldsymbol{g})$ is

$$
\left.\frac{d}{d s}\right|_{s=0} K\left(\boldsymbol{r}, \boldsymbol{p} ; \boldsymbol{g}_{s}\right)=-\sum_{\alpha=1}^{N} \frac{1}{2 m^{\alpha}} \int_{\Omega} h^{k l}(\boldsymbol{x}) p_{k}^{\alpha} p_{l}^{\alpha} \delta\left(\boldsymbol{x}-\boldsymbol{r}^{\alpha}\right) d \Omega,
$$

where we have used the identity

$$
\left.\frac{d}{d s}\right|_{s=0} g_{s}^{i j}(\boldsymbol{x})=-g_{0}^{i k}(\boldsymbol{x}) h_{k l}(\boldsymbol{x}) g_{0}^{l j}(\boldsymbol{x})=-h^{i j}(\boldsymbol{x}) .
$$

Recalling Eq. (28), it is clear that Eq. (38) allows us to identify the functional derivative as

$$
\frac{\delta K}{\delta g_{i j}}(\boldsymbol{x})=-\sum_{\alpha=1}^{N} \frac{1}{2 m^{\alpha}} g_{0}^{k i}(\boldsymbol{x}) g_{0}^{j l}(\boldsymbol{x}) p_{k}^{\alpha} p_{l}^{\alpha} \delta\left(\boldsymbol{x}-\boldsymbol{r}^{\alpha}\right) .
$$

Noting that $m^{\alpha} v^{\alpha i}=g_{0}^{i k}\left(\boldsymbol{r}^{\alpha}\right) p_{k}^{\alpha}$ and taking the ensemble average, we reach the classical Irving-Kirkwood result

$$
\sigma_{K}^{i j}(\boldsymbol{x})=-\sum_{\alpha=1}^{N} m^{\alpha}\left\langle v^{\alpha i} v^{\alpha j} \delta\left(\boldsymbol{x}-\boldsymbol{r}^{\alpha}\right)\right\rangle .
$$

We discuss next the uniqueness of the kinetic stress. Because the kinetic energy can be written as the integral of a kinetic energy density

$$
K=\int_{\Omega} k\left(\boldsymbol{x} ; \boldsymbol{g}_{s}(\boldsymbol{x})\right) d \Omega
$$

which depends locally on the metric tensor,

$$
k\left(\boldsymbol{x} ; \boldsymbol{g}_{s}(\boldsymbol{x})\right)=\sum_{\alpha=1}^{N} \frac{1}{2 m^{\alpha}} g_{s}^{i j}(\boldsymbol{x}) p_{i}^{\alpha} p_{j}^{\alpha} \delta\left(\boldsymbol{x}-\boldsymbol{r}^{\alpha}\right),
$$


then the functional derivative can be localized to the partial derivative and

$$
\boldsymbol{\sigma}_{K}(\boldsymbol{x})=2\left\langle\frac{\partial k}{\partial \boldsymbol{g}}(\boldsymbol{x})\right\rangle .
$$

This formula leads directly to Eq. (41) and is devoid of ambiguity. The key observation in this argument is the existence of a local energy density.

\subsection{Potential part of the microscopic stress}

We focus now on the potential contribution to the stress tensor

$$
\boldsymbol{\sigma}_{V}=2\left\langle\frac{\delta V}{\delta \boldsymbol{g}}\right\rangle .
$$

Unfortunately, the potential energy cannot be naturally expressed as the integral of a potential energy density, i.e. there is no canonical notion of how to localize in space the potential energy of a set of discrete interacting particles (Admal and Tadmor, 2011). However, the cluster expansion in Eq. (8) provides a systematic way to localize as much as possible the potential interactions. By computing variations for each cluster potential $V_{I_{n}}$ independently, we partially localize these variations because they are only affected by changes of metric that alter the distances between the particles involved, but are independent of changes of metric that alter other regions of space.

The significance of Eq. (26) is that we can compute variations of $A$ (or of $V$ ) by keeping the particle positions fixed and just changing the background metric $\boldsymbol{g}_{s}$ as given by Eqs. $(20,27)$. Therefore, we can write the inter-particle distances as

$$
r^{\alpha \beta}(s)=\int_{0}^{1} \sqrt{\left[g_{s}\right]_{i j}(\boldsymbol{c}(\lambda, s)) \partial_{\lambda} c^{i}(\lambda, s) \partial_{\lambda} c^{j}(\lambda, s)} d \lambda,
$$


where now the curve $\boldsymbol{c}(\lambda, s)$ is the geodesic relative to $\boldsymbol{g}_{s}$ joining two particles with fixed coordinates, and consequently we can write the potential energy as

$$
V(s)=\sum_{n=2}^{N} \sum_{I_{n}=1}^{M_{n}} \widetilde{V}_{I_{n}} \circ R_{I_{n}}(s),
$$

where $R_{I_{n}}$ maps the parameter $s$ to the sets of distances $\left\{r^{\alpha \beta}\right\}$ involved in the potential contribution $\widetilde{V}_{I_{n}}$ measured with metric tensor $\boldsymbol{g}_{s}$. Because we only consider metric tensors defined as in Eq. (20), the distances produced by $R_{I_{n}}$ lie on the shape space $\mathcal{S}_{I_{n}}$, i.e. the range of $R_{I_{n}}: \mathbb{R}_{+} \longrightarrow \mathcal{S}_{I_{n}}$ is precisely the domain of $\widetilde{V}_{I_{n}}: \mathcal{S}_{I_{n}} \longrightarrow \mathbb{R}$. For this reason, potential extensions are not necessary in the present framework.

To identify $\delta V / \delta \boldsymbol{g}$ in Eq. (45), we need to evaluate the derivative of $V$ with respect to $s$. A crucial observation is that the chain rule applied to Eq. (47) naturally involves the tangent map of $\widetilde{V}_{I_{n}}$, which being $\widetilde{V}_{I_{n}}$ a scalar function is homeomorphic to the covariant derivative of $\widetilde{V}_{I_{n}}$ along $\mathcal{S}_{I_{n}}$. Thus, we obtain

$$
\left.\frac{d}{d s}\right|_{s=0} V=\left.\sum_{n=2}^{N} \sum_{I_{n}=1}^{M_{n}} \sum_{\alpha, \beta>\alpha} \varphi_{\alpha \beta}^{\mathcal{S}_{I_{n}}} \frac{d}{d s}\right|_{s=0} r^{\alpha \beta}(s)
$$

where, in contrast to Eq. (7), here

$$
\varphi_{\alpha \beta}^{\mathcal{S}_{I_{n}}}=\left(\nabla_{\mathcal{S}_{I_{n}}} \tilde{V}_{I_{n}}\right)_{\alpha \beta}
$$

stands for the $\alpha \beta$ component of the covariant derivative of the potential along the shape space $\mathcal{S}_{I_{n}}$ expressed in the canonical basis of $\mathcal{D}_{I_{n}}$. Because the coordinates $\left\{r^{\alpha \beta}\right\}$ do not parametrize $\mathcal{S}_{I_{n}}$ but rather its embedding space $\mathcal{D}_{I_{n}}$, the covariant derivative can be understood as the projection onto $\mathcal{S}_{I_{n}}$ of the gradient of a potential extension in $\mathcal{D}_{I_{n}}$. The result is however independent 
of the extension. From a different rationale, the projection of the CFD onto shape space has been recently proposed in analogy with the Beltrami decomposition of symmetric tensors (Admal, 2014).

Further elaborating on Eq. (48), we have

$$
\begin{aligned}
\left.\frac{d}{d s}\right|_{s=0} V & =\sum_{n=2}^{N} \sum_{I_{n}=1}^{M_{n}} \sum_{\alpha, \beta>\alpha} \varphi_{\alpha \beta}^{\mathcal{S}_{I_{n}}} \int_{\Omega} \frac{\delta r^{\alpha \beta}}{\delta g_{i j}} h_{i j} d \Omega \\
& =\int_{\Omega} \sum_{n=2}^{N} \sum_{I_{n}=1}^{M_{n}} \sum_{\alpha, \beta>\alpha} \varphi_{\alpha \beta}^{\mathcal{S}_{I_{n}}} \frac{\delta r^{\alpha \beta}}{\delta g_{i j}} h_{i j} d \Omega,
\end{aligned}
$$

which recalling Eq. (30) leads to

$$
\sigma_{V, \text { inst }}^{i j}(\boldsymbol{x})=2 \sum_{n=2}^{N} \sum_{I_{n}=1}^{M_{n}} \sum_{\alpha, \beta>\alpha} \varphi_{\alpha \beta}^{\mathcal{S}_{I_{n}}} \frac{\delta r^{\alpha \beta}}{\delta g_{i j}}(\boldsymbol{x}) .
$$

To evaluate this expression, we use Eq. (46) to compute

$$
\begin{aligned}
& \left.\frac{d}{d s}\right|_{s=0} r^{\alpha \beta}=\int_{\Omega} \frac{\delta r^{\alpha \beta}}{\delta g_{i j}} h_{i j} d \Omega \\
& \quad=\int_{0}^{1} \frac{1}{2\left|\partial_{\lambda} c_{0}\right|_{g_{0}}}\left\{h_{i j} \partial_{\lambda} c_{0}^{i} \partial_{\lambda} c_{0}^{j}+D_{s} g_{i j} \partial_{\lambda} c_{0}^{i} \partial_{\lambda} c_{0}^{j}+2 g_{i j} D_{s} \partial_{\lambda} c_{0}^{i} \partial_{\lambda} c_{0}^{j}\right\} d \lambda,
\end{aligned}
$$

where $\boldsymbol{c}_{0}(\lambda)=\boldsymbol{c}(\lambda, 0)$ and $D_{s}$ denotes the covariant differentiation along the vector field $\partial_{s} \boldsymbol{c}(\lambda, 0)$ (Do Carmo, 1992). See Yavari and Marsden (2009) for a related calculation when examining energy invariance of particle systems in a Riemannian manifold. Here, the variation of the length does not involve boundary terms because the end points of the geodesic do not depend on $s$. The second term of the integrand vanishes because the covariant derivative of the metric tensor is zero. To treat the last term, we first use the fact that $D_{s} \partial_{\lambda} \boldsymbol{c}=D_{\lambda} \partial_{s} \boldsymbol{c}$ (Do Carmo, 1992). Then, it is easily seen that it vanishes. Indeed,

$$
\int_{0}^{1} \frac{1}{\left|\partial_{\lambda} \boldsymbol{c}_{0}\right|_{\boldsymbol{g}_{0}}} g_{i j} D_{\lambda} W^{i} \partial_{\lambda} c_{0}^{j} d \lambda
$$


is the variation of the length of $\boldsymbol{c}_{0}$ with respect to a variation of this curve along the vector field $\boldsymbol{W}$. Because $\boldsymbol{c}_{0}$ is a geodesic, this expression vanishes for all $\boldsymbol{W}$ vanishing at the ends of the curve, in particular $\partial_{s} \boldsymbol{c}$.

Thus, retaining only the first term we can express the variation of bond lengths in terms of an integral over the whole space by resorting to a Dirac distribution

$$
\left.\frac{d}{d s}\right|_{s=0} r^{\alpha \beta}(s)=\int_{\Omega} \int_{0}^{1} \delta\left(\boldsymbol{c}_{0}(\lambda)-\boldsymbol{x}\right) \frac{h_{i j}(\boldsymbol{x}) \partial_{\lambda} c_{0}^{i} \partial_{\lambda} c_{0}^{j}}{2 \sqrt{\left[g_{0}\right]_{k l}(\boldsymbol{x}) \partial_{\lambda} c_{0}^{k} \partial_{\lambda} c_{0}^{l}}} d \lambda d \Omega
$$

which allows us to identify the functional derivative as

$$
\frac{\delta r^{\alpha \beta}}{\delta g_{i j}}(\boldsymbol{x})=\int_{0}^{1} \delta\left(\boldsymbol{c}_{0}(\lambda)-\boldsymbol{x}\right) \frac{\partial_{\lambda} c_{0}^{i} \partial_{\lambda} c_{0}^{j}}{2 \sqrt{\left[g_{0}\right]_{k l}(\boldsymbol{x}) \partial_{\lambda} c_{0}^{k} \partial_{\lambda} c_{0}^{l}}} d \lambda .
$$

Considering Cartesian coordinates $\left(\left[g_{0}\right]_{i j}=\delta_{i j}\right)$ and parametrizing the straight line as $\boldsymbol{c}_{0}(\lambda)=(1-\lambda) \boldsymbol{r}^{\alpha}+\lambda \boldsymbol{r}^{\beta}$, this expression simplifies to

$$
\frac{\delta r^{\alpha \beta}}{\delta \boldsymbol{g}}(\boldsymbol{x})=\frac{1}{2} \frac{\boldsymbol{r}^{\alpha \beta} \otimes \boldsymbol{r}^{\alpha \beta}}{r^{\alpha \beta}} \underbrace{\int_{0}^{1} \delta\left[(1-\lambda) \boldsymbol{r}^{\alpha}+\lambda \boldsymbol{r}^{\beta}-\boldsymbol{x}\right]}_{B\left(\boldsymbol{r}^{\alpha}, \boldsymbol{r}^{\beta} ; \boldsymbol{x}\right)} d \lambda .
$$

Recalling Eq. (52), we obtain an expression for the potential part of the stress tensor

$$
\begin{aligned}
\boldsymbol{\sigma}_{V}(\boldsymbol{x}) & =\sum_{n=2}^{N} \sum_{I_{n}=1}^{M_{n}} \sum_{\alpha, \beta>\alpha}\left\langle\varphi_{\alpha \beta}^{\mathcal{S}_{I_{n}}} \frac{\boldsymbol{r}^{\alpha \beta} \otimes \boldsymbol{r}^{\alpha \beta}}{r^{\alpha \beta}} B\left(\boldsymbol{r}^{\alpha}, \boldsymbol{r}^{\beta} ; \boldsymbol{x}\right)\right\rangle \\
& =\left\langle\sum_{\alpha, \beta>\alpha} \boldsymbol{f}^{\alpha \beta} \otimes \boldsymbol{r}^{\alpha \beta} B\left(\boldsymbol{r}^{\alpha}, \boldsymbol{r}^{\beta} ; \boldsymbol{x}\right)\right\rangle,
\end{aligned}
$$

which, remarkably, is the Irving-Kirkwood result with a force decomposition

$$
\boldsymbol{f}^{\alpha \beta}=\sum_{n=2}^{N} \sum_{I_{n}=1}^{M_{n}} \varphi_{\alpha \beta}^{\mathcal{S}_{I_{n}}} \hat{\boldsymbol{r}}^{\alpha \beta}
$$


This last expression should be understood as a sum over all potential contributions that involve particles $\alpha$ and $\beta$. This covariant central force decomposition (cCFD) is a close analog of the usual CFD in Eq. (6), which replaces the partial differentiation of $\widetilde{V}_{I_{n}}$ by a covariant differentiation along the shape spaces $\mathcal{S}_{I_{n}}$. Since $\mathcal{S}_{I_{n}}$ is an open subset of $\mathcal{D}_{I_{n}}$ for $n \leq 4$, cCFD and CFD coincide in this case. However, when $n>5$, our definition resolves the ambiguity of the usual CFD.

\section{Evaluating the covariant derivative of the potential along the shape space}

Practically, the evaluation of $\left(\nabla_{\mathcal{S}_{I_{n}}} \widetilde{V}_{I_{n}}\right)_{\alpha \beta}$ can be performed by first computing the gradient of an extension of the potential in the distance space $\mathcal{D}_{I_{n}}, \nabla_{\mathcal{D}_{I_{n}}} \widetilde{V}_{I_{n}}$, and then projecting the result onto the tangent of the shape space $\mathcal{S}_{I_{n}}$.

The calculation of $\nabla_{\mathcal{D}_{I_{n}}} \widetilde{V}_{I_{n}}$, see Eq. (10), for an arbitrary extension can be performed by solving the following linear system of equations

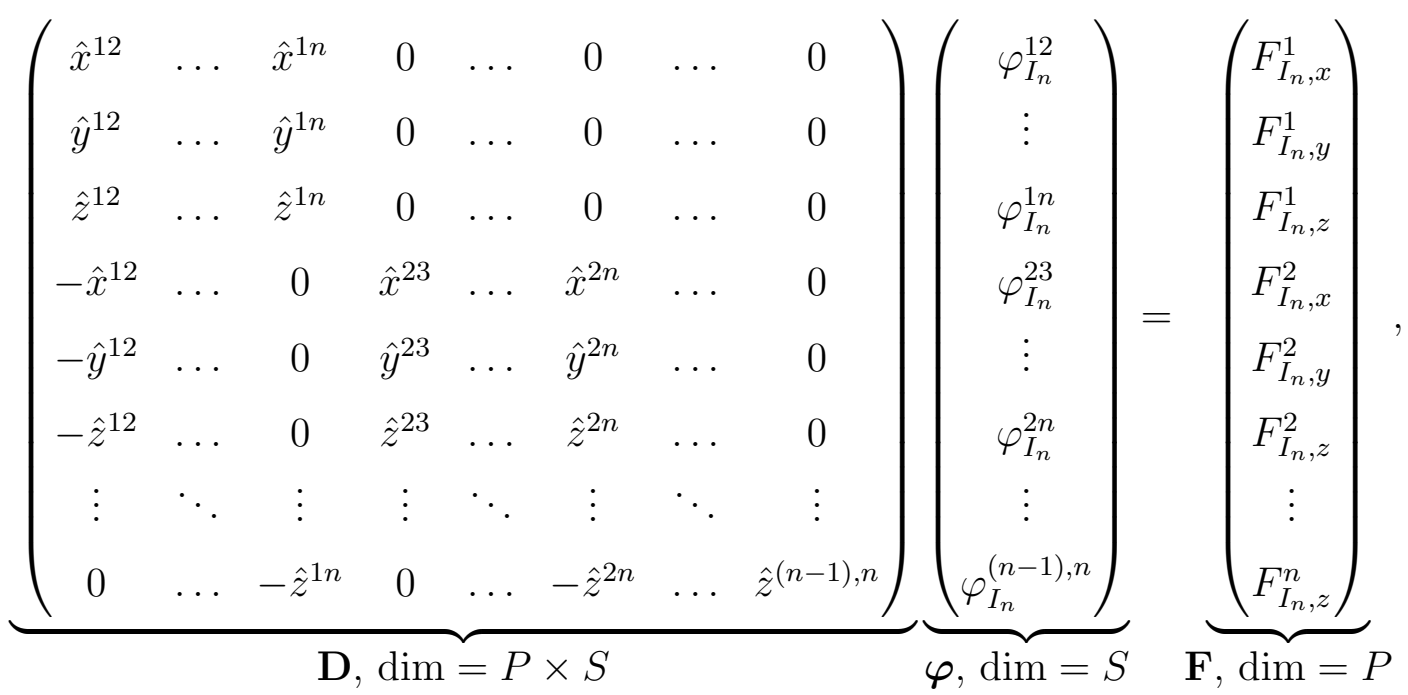


which reflects the fact that $\boldsymbol{F}_{I_{n}}^{\alpha}=-\partial V_{I_{n}} / \partial \boldsymbol{r}^{\alpha}=\sum_{\beta} \varphi_{\alpha \beta}^{I_{n}} \hat{\boldsymbol{r}}^{\alpha \beta}$. Here, $P=$ $3 n, S=n(n-1) / 2$, and the rank of $\mathbf{D}$ is $R=3 n-6$ (the number of degrees of freedom of a set of $n$ particles satisfying balance of linear and angular momentum). A particular solution of this system can be obtained, for instance, by minimizing the norm of the solution $\varphi$.

We now note that the normal space to the shape space $\mathcal{S}_{I_{n}}$ is precisely the kernel of $\mathbf{D}$, i.e. it is the vector space spanned by the solutions of

$$
\mathrm{DX}=\mathbf{0} .
$$

Any component of the force decomposition on this space does not alter the net forces on the particles, as can be checked by comparing Eqs. (60) and (61). The solution to this problem can be computed through a QR decomposition. Let $\mathbf{D}^{T}$ be the transpose of $\mathbf{D}$. Its QR decomposition exists and has the general form

$$
\mathbf{D}^{T} \mathbf{P}=\mathbf{Q R}=\left(\begin{array}{ll}
\mathbf{Q}_{1} & \mathbf{Q}_{2}
\end{array}\right)\left(\begin{array}{cc}
\mathbf{R}_{1} & \mathbf{R}_{2} \\
\mathbf{0} & \mathbf{0}
\end{array}\right)
$$

where $\mathbf{Q}$ is an orthogonal matrix of dimension $S \times S, \mathbf{Q}_{1}$ is a $S \times R$ matrix with orthonormal columns (this is uniquely defined), $\mathbf{Q}_{2}$ is a $S \times(S-R)$ matrix with orthonormal columns, $\mathbf{R}_{1}$ is a $R \times R$ upper triangular and invertible matrix, $\mathbf{R}_{2}$ is a $R \times(P-R)$ matrix, $\mathbf{P}$ is a $P \times P$ pivoting matrix. Then we can rewrite Eq. (61) as

$$
\mathbf{D X}=\mathbf{P R}^{T} \mathbf{Q}^{T} \mathbf{X}=\mathbf{P}\left(\begin{array}{ll}
\mathbf{R}_{1}^{T} & \mathbf{0} \\
\mathbf{R}_{2}^{T} & \mathbf{0}
\end{array}\right)\left(\begin{array}{c}
\mathbf{Q}_{1}^{T} \\
\mathbf{Q}_{2}^{T}
\end{array}\right) \mathbf{X}=\mathbf{0} .
$$

Taking into account that $\mathbf{Q}$ is an orthogonal matrix, its columns form an orthonormal basis of $\mathbb{R}^{S}$. We can then define the two components of $\mathbf{X}$ on the 
subspaces spanned by the columns of $\mathbf{Q}_{1}$ and $\mathbf{Q}_{2}, \mathbf{X}_{1}=\mathbf{Q}_{1}^{T} \mathbf{X}, \mathbf{X}_{2}=\mathbf{Q}_{2}^{T} \mathbf{X}$. Inserting this decomposition in Eq. (63) we obtain the equivalent system

$$
\left(\begin{array}{l}
\mathbf{R}_{1}^{T} \mathbf{X}_{1} \\
\mathbf{R}_{2}^{T} \mathbf{X}_{1}
\end{array}\right)=\mathbf{0} .
$$

Since $\mathbf{R}_{1}^{T}$ is invertible, this results in $\mathbf{X}_{1}=\mathbf{0}$, while the component $\mathbf{X}_{2}$ is completely free. In other words, the kernel of $\mathbf{D}$ is the subspace formed by the column vectors of $\mathrm{Q}_{2}$. Therefore, given a $\mathrm{CFD} \boldsymbol{\varphi}$, its projection onto the shape space is simply

$$
\varphi_{\mathcal{S}_{I_{n}}}=\mathrm{Q}_{1} \mathrm{Q}_{1}^{T} \boldsymbol{\varphi}
$$

An alternative method to compute the cCFD involves Caley-Menger determinants. As illustration, let us examine the simplest example of the cCFD for a 5-body potential following this methodology. In this case, $\mathcal{D}_{I_{n}}=\mathbb{R}_{+}^{10}$ while $\mathcal{S}_{I_{n}}$ is a hypersurface with dimension $(3 \cdot 5-6)=9$, where 6 stands for the rigid body degrees of freedom. It can be shown (Tadmor and Miller, 2011) that $\mathcal{S}_{I_{n}}$ can be locally characterized by the equation

$$
\chi=\operatorname{det}\left(\begin{array}{cccccc}
0 & s^{12} & s^{13} & s^{14} & s^{15} & 1 \\
s^{12} & 0 & s^{23} & s^{24} & s^{25} & 1 \\
s^{13} & s^{23} & 0 & s^{34} & s^{35} & 1 \\
s^{14} & s^{24} & s^{34} & 0 & s^{45} & 1 \\
s^{15} & s^{25} & s^{35} & s^{45} & 0 & 1 \\
1 & 1 & 1 & 1 & 1 & 0
\end{array}\right)=0
$$

where $\chi$ is a Caley-Menger determinant and $s^{\alpha \beta}=\left(r^{\alpha \beta}\right)^{2}$. The normal to 
$\mathcal{S}_{I_{n}}$ can then be computed as

$$
\begin{aligned}
\boldsymbol{n}_{\mathcal{S}_{I_{n}}} & =\left[\left(\nabla_{\mathcal{D}_{I_{n}}} \chi\right)_{\alpha \beta}\right]=\left(\frac{\partial \chi}{\partial r^{12}}, \ldots, \frac{\partial \chi}{\partial r^{45}}\right), \\
\widehat{\boldsymbol{n}}_{\mathcal{S}_{I_{n}}} & =\frac{\boldsymbol{n}_{\mathcal{S}_{I_{n}}}}{\left\|\boldsymbol{n}_{\mathcal{S}_{I_{n}}}\right\|}
\end{aligned}
$$

Thus, we can evaluate the covariant derivative of the potential along $\mathcal{S}_{I_{n}}$ by projecting the derivative of an extension of the potential onto the tangent space using the normal, i.e.

$$
\begin{aligned}
& \left(\nabla_{\mathcal{S}_{I_{n}}} \widetilde{V}_{I_{n}}\right)_{\alpha \beta}=\frac{\partial \widetilde{V}_{I_{n}}}{\partial r^{\alpha \beta}}-\left(\nabla_{\mathcal{D}_{I_{n}}} \widetilde{V}_{I_{n}} \cdot \widehat{\boldsymbol{n}}_{\mathcal{S}_{I_{n}}}\right)\left(\widehat{n}_{\mathcal{S}_{I_{n}}}\right)_{\alpha \beta}= \\
& =\frac{\partial V_{\alpha \beta}}{\partial r^{\alpha \beta}}-\frac{\left(\widehat{n}_{\mathcal{S}_{I_{n}}}\right)_{\alpha \beta}}{\left\|\boldsymbol{n}_{\mathcal{S}_{I_{n}}}\right\|^{2}}\left(\frac{\partial \widetilde{V}_{I}}{\partial r^{12}}, \ldots, \frac{\partial \widetilde{V}_{I}}{\partial r^{45}}\right)\left(\begin{array}{c}
\frac{\partial \chi}{\partial r^{12}} \\
\vdots \\
\frac{\partial \chi}{\partial r^{45}}
\end{array}\right) .
\end{aligned}
$$

This is equivalent to the QR method presented before (the normal in Eq. (67) is the generator of the null space of $\mathbf{D})$. To exercise this formula, we consider the following 5-body potential

$$
\begin{gathered}
\widetilde{V}_{I_{n}}\left(r^{12}, \ldots, r^{45}\right)=\left(s^{12}+3 s^{13}+s^{14}+2 s^{15}+5 s^{23}+2 s^{24}\right. \\
\left.+5 s^{25}+s^{34}+s^{35}+4 s^{45}\right)^{1 / 2}
\end{gathered}
$$

We want to evaluate the force decomposition Eq. (59) at positions

$$
\begin{aligned}
& \boldsymbol{r}^{1}=(0,0,0), \quad \boldsymbol{r}^{2}=(1,0,0), \\
& \boldsymbol{r}^{3}=(1,0,1), \quad \boldsymbol{r}^{4}=(0,1,-2), \\
& \boldsymbol{r}^{5}=(-2,1,3) .
\end{aligned}
$$


The normal vector to $\mathcal{S}_{I_{n}}$ at this configuration is

$$
\left[\boldsymbol{n}_{S_{I_{n}}}\right]=\left(\begin{array}{c}
-0.234 \\
0.236 \\
0.075 \\
-0.125 \\
-0.585 \\
-0.287 \\
0.510 \\
0.277 \\
-0.313 \\
-0.090
\end{array}\right)
$$

and the gradient of the potential, if we consider its trivial extension to $\mathcal{D}_{I_{n}}$, is

$$
\left[\varphi_{\alpha \beta}^{I_{n}}\right]=\left[\left(\nabla_{\mathcal{D}_{I_{n}}} \widetilde{V}_{I_{n}}\right)_{\alpha \beta}\right]=\left(\begin{array}{c}
0.058 \\
0.248 \\
0.131 \\
0.437 \\
0.292 \\
0.286 \\
1.273 \\
0.194 \\
0.219 \\
1.258
\end{array}\right) .
$$


Therefore, we obtain the covariant derivative

$$
\left[\varphi_{\alpha \beta}^{\mathcal{S}_{I_{n}}}\right]=\left[\left(\nabla_{\mathcal{S}_{I_{n}}} \widetilde{V}_{I_{n}}\right)_{\alpha \beta}\right]=\left(\begin{array}{c}
0.121 \\
0.184 \\
0.111 \\
0.471 \\
0.449 \\
0.363 \\
1.136 \\
0.119 \\
0.303 \\
1.283
\end{array}\right) .
$$

Eq. (72) is used in the standard CFD, while Eq. (73) appears in the cCFD. The two arrays are significantly different. If we now take another extension of the potential to the distance space, summing for instance the Caley-Menger determinant in Eq. (66) to the potential $\widetilde{V}_{I_{n}}$ (which does not affect the potential along $\mathcal{S}_{I_{n}}$ as the Caley-Menger determinant is 0 on it), we obtain 


$$
\left[\varphi_{\alpha \beta}^{I_{n}}\right]=\left[\left(\nabla_{\mathcal{D}_{I_{n}}} \widetilde{V}_{I_{n}}\right)_{\alpha \beta}\right]=\left(\begin{array}{c}
-447.942 \\
452.796 \\
143.239 \\
-239.0289 \\
-1119.708 \\
-548.399 \\
977.667 \\
530.854 \\
-598.447 \\
-171.067
\end{array}\right)
$$

while the $\mathrm{cCDF}$ result

$$
\left[\varphi_{\alpha \beta}^{\mathcal{S}_{I_{n}}}\right]=\left[\left(\nabla_{\mathcal{S}_{I_{n}}} \widetilde{V}_{I_{n}}\right)_{\alpha \beta}\right]=\left(\begin{array}{c}
0.121 \\
0.184 \\
0.111 \\
0.471 \\
0.449 \\
0.363 \\
1.136 \\
0.119 \\
0.303 \\
1.283
\end{array}\right),
$$

is independent of the representation of the potential in terms of distances between particles. Thus, this example shows that, while CFD is extensiondependent, cCFD is uniquely defined as the covariant derivative of the potential along the shape space $\mathcal{S}_{I_{n}}$. 


\section{Numerical results}

In this section we exercise the theory described previously on a coiledcoil structural protein. This protein is composed of two identical $\alpha$-helical chains that wrap around each other to form a super-helix (see Fig. 1A). The inner core of this coiled-coil is composed of intercalating hydrophobic amino acids, and is surrounded by opposing negatively and positively charged amino acids. We consider an infinitely long protein, modeled with periodic boundary conditions. The interatomic potential is taken from a widely used protein force-field (CHARMM22/CMAP), which comprises 2- (bonds, Coulomb and van der Waals interactions), 3- (angle potentials), 4- (torsional potentials) and 5-body (cross-term energy correction map, CMAP) interactions. The system is simulated in a NVT ensemble, with a fixed simulation box and the temperature held constant at $298 \mathrm{~K}$. More details on the simulation procedure can be found in Torres-Sánchez et al. (2015).

As previously mentioned, 2-, 3- and 4-body interactions are straightforward to decompose in a CFD: once the net forces $\boldsymbol{F}^{\alpha}$ are computed, the system of equations in Eq. (60) admits a unique solution for the pairwise terms $\boldsymbol{f}^{\alpha \beta}$. In this case both CFD and cCFD give the same results. In contrast, Eq. (60) for 5-body interactions such as CMAP admits infinitely many solutions. The CMAP interaction (MacKerell et al., 2004) is a backbone correction that depends on two dihedral angles, $\phi$ and $\psi$. In the $\phi \psi$-plane, values of the potential are given on a grid, which are then interpolated. Since dihedral angles can be expressed in terms of distances, we can give a natural extension of the potential $V_{\mathrm{CMAP}}\left(\phi\left(\left\{r^{\alpha \beta}\right\}\right), \psi\left(\left\{r^{\alpha \beta}\right\}\right)\right)$, where $\phi$ depends on the distances between the first four particles, while $\psi$ depends on the 
distances between the last four particles. A natural CFD follows

$$
\varphi_{\alpha \beta}=\frac{\partial V_{\mathrm{CMAP}}(\phi, \psi)}{\partial \phi} \frac{\partial \phi}{\partial r^{\alpha \beta}}+\frac{\partial V_{\mathrm{CMAP}}(\phi, \psi)}{\partial \psi} \frac{\partial \psi}{\partial r^{\alpha \beta}} .
$$

With the terminology of Admal and Tadmor (2010), this CFD corresponds to a particular extension of the CMAP potential. This CFD, or any other CFD, can then be projected onto $\mathcal{S}_{I_{n}}$ to obtain the cCFD. However, as described in Section 4, a solution to Eq. (60) can be found without introducing an extension by minimizing the norm of $\boldsymbol{\varphi}$. This latter method has two major advantages. First, it is less intrusive with respect to the MD code. This is because it only requires the net forces acting on the particles, which are directly provided by the MD code, rather than the partial derivatives in Eq. (76), which would require modifying the CMAP routine. Second, this method is completely general for any n-body potential, and does not rely on its specific functional form.

To analyze the microscopic stress in the coiled-coil protein, we adopt the methodology presented in Torres-Sánchez et al. (2015); Vanegas and Arroyo (2014) and plot the traction

$$
\boldsymbol{t}=\boldsymbol{\sigma} \cdot \boldsymbol{n}
$$

on the external surface of the protein, where $\boldsymbol{n}$ stands for the outward normal to the surface. This surface is determined from a level set of the mass density of the protein. We then separate the normal traction, $t_{n}=\boldsymbol{t} \cdot \boldsymbol{n}$, which we represent as a color map, and the tangential traction, $\boldsymbol{\tau}=\boldsymbol{t}-t_{n} \boldsymbol{n}$, which we represent with arrows. Both the density and the stress are ensemble averaged and smoothed by means of a Gaussian filter. 


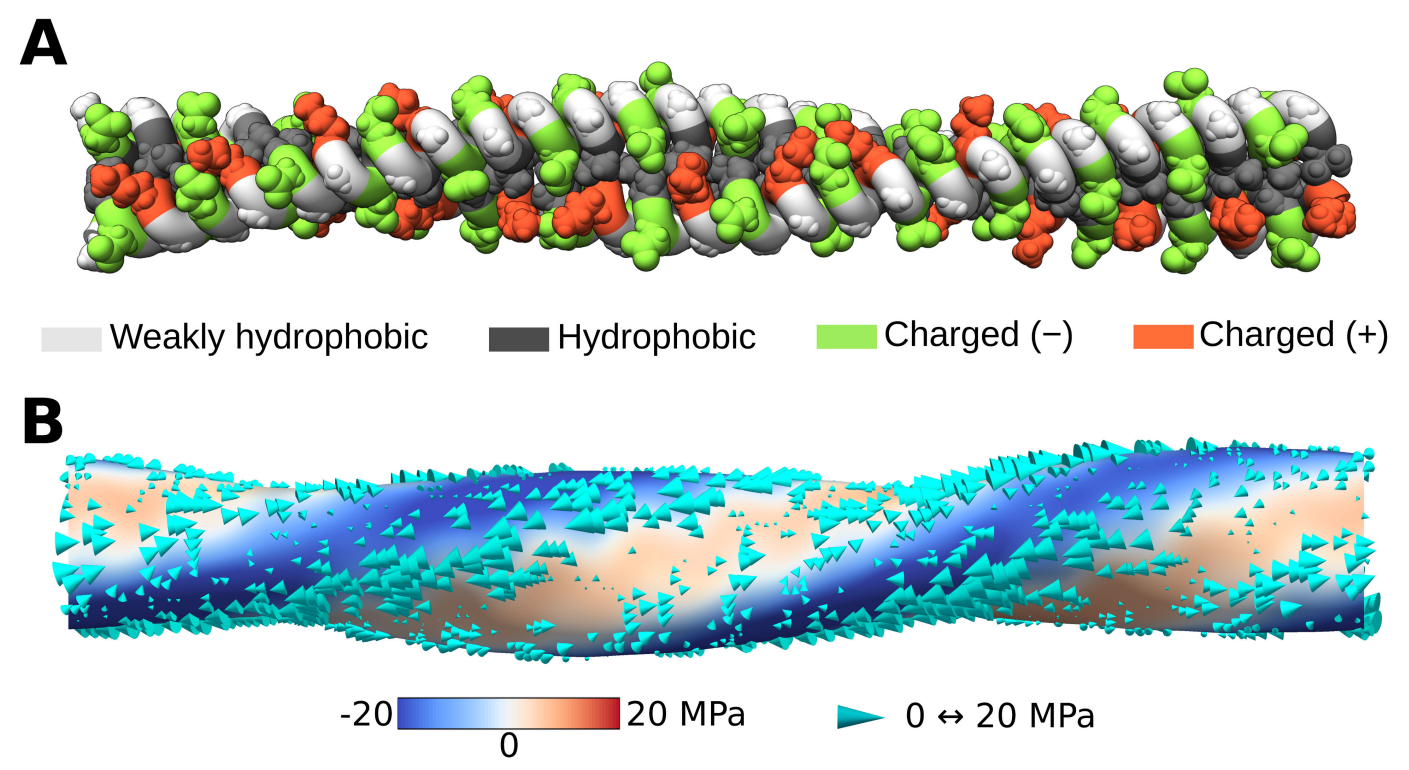

Figure 1: Structure of the coiled-coil protein (A) and total traction $\boldsymbol{t}=\boldsymbol{\sigma} \cdot \boldsymbol{n}$ on the coiled-coil (B). The total traction is split into the normal traction $t_{n}=\boldsymbol{t} \cdot \boldsymbol{n}$, which is represented as a color map, and the lateral traction $\boldsymbol{\tau}$, which we plot with arrows. 


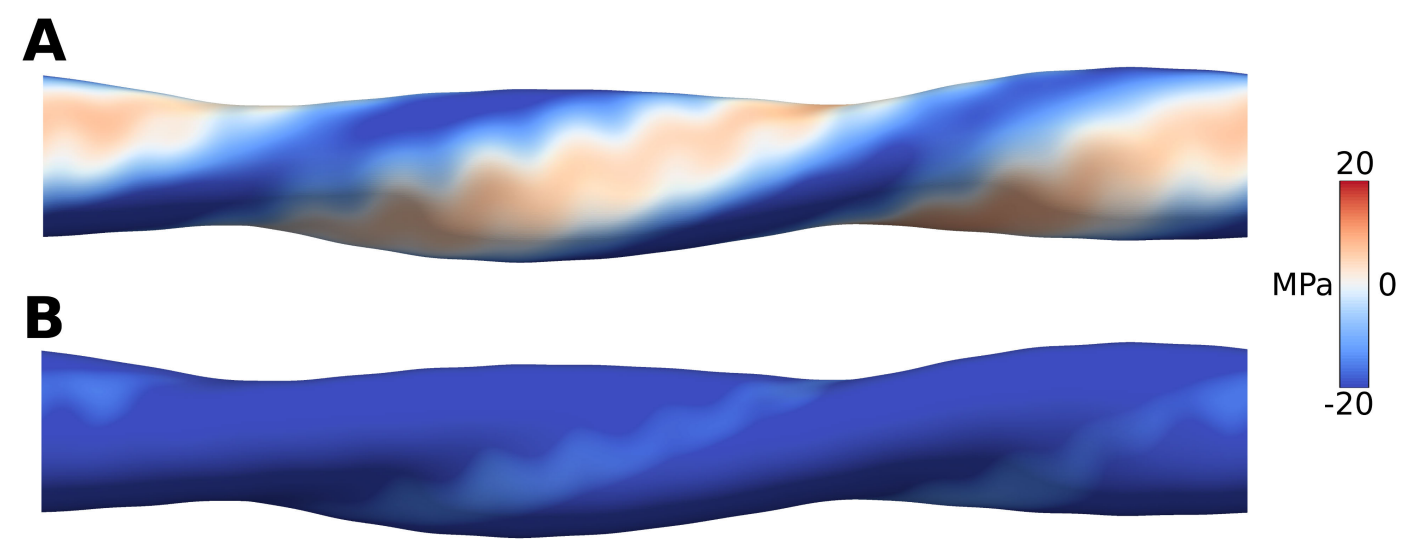

FiguRE 2: Normal traction on the surface of the coiled-coil protein for the total stress (A) and for the stress without the contribution from the CMAP interaction (B).

In Fig. 1B we plot the traction for a Gaussian filter of standard deviation $0.4 \mathrm{~nm}$. From this Figure we can extract two major conclusions. First, we observe that the zippered interface between the two coils produces outward tractions (red), while the periphery of the protein is dominated by inward tractions (blue). Regarding $\boldsymbol{\tau}$, we observe that tractions are larger at the periphery of the protein with opposite regions where arrows go leftwards and rightwards respectively. The total force and torque obtained as surface integrals of the traction are negligible since the cCDF stress is in mechanical equilibrium.

Since the CMAP interaction is a higher-body correction that complicates the calculation of the microscopic stress, one may be tempted to simply ignore it in the analysis of the MD trajectory. To examine this, we plot the normal traction on the protein surface considering all interactions and following the cCFD proposed here (Fig. 2A) and the traction obtained ignoring the CMAP contributions to the microscopic stress Fig. 2B. The figure clearly shows 
that the CMAP contribution is very significant and cannot be ignored, since otherwise the normal traction is only inwards. This highlights the importance of properly dealing with higher-order interactions, as cCFD does.

We now examine the differences between cCFD and different CFDs when analyzing the CMAP potential in the coiled-coil (Fig. 3). We first we compare cCFD (A) with CFD in Eq. (76) (B) and see small, yet not negligible, differences $(\mathrm{C})$. To highlight the effect of the extension on the resulting CFD, we examine an alternative extension of the potential of the form $V_{\mathrm{CMAP}}+k \chi$, where $\chi$ is the Caley-Menger determinant defined in Eq. (66) (D). We see that in this case, due to the effect of the Caley-Menger determinant, contributions of the resulting CFD along the normal to $\mathcal{S}$ distort the stress field, leading to tractions that differ from those of cCFD largely. We finally compute the CFD obtained from the solution to Eq. (60) that minimizes the norm of the CFD (D). This CFD, which does not have a clear physical justification, results in a very non-homogeneous stress, which lacks of a meaningful interpretation. It is remarkable that cCFD is obtained from this CFD by projecting onto $\mathcal{S}$. Thus, selecting the extension of the potential for a CFD is a delicate subject and can lead to very different stresses, some of which do not have a clear physical interpretation. On the other hand, cCFD is independent of the extension of the potential and provides physically meaningful stresses.

We next use this example to examine the interpretation of the microscopic stress in such a nanoscale mechanical system. For this, we represent in Fig. 4 the structure and stress field with different levels of resolution as given by the standard deviation of the Gaussian filter. For a Gaussian filter of standard deviation of $0.1 \mathrm{~nm}$ for both the stress and the density (A) we 

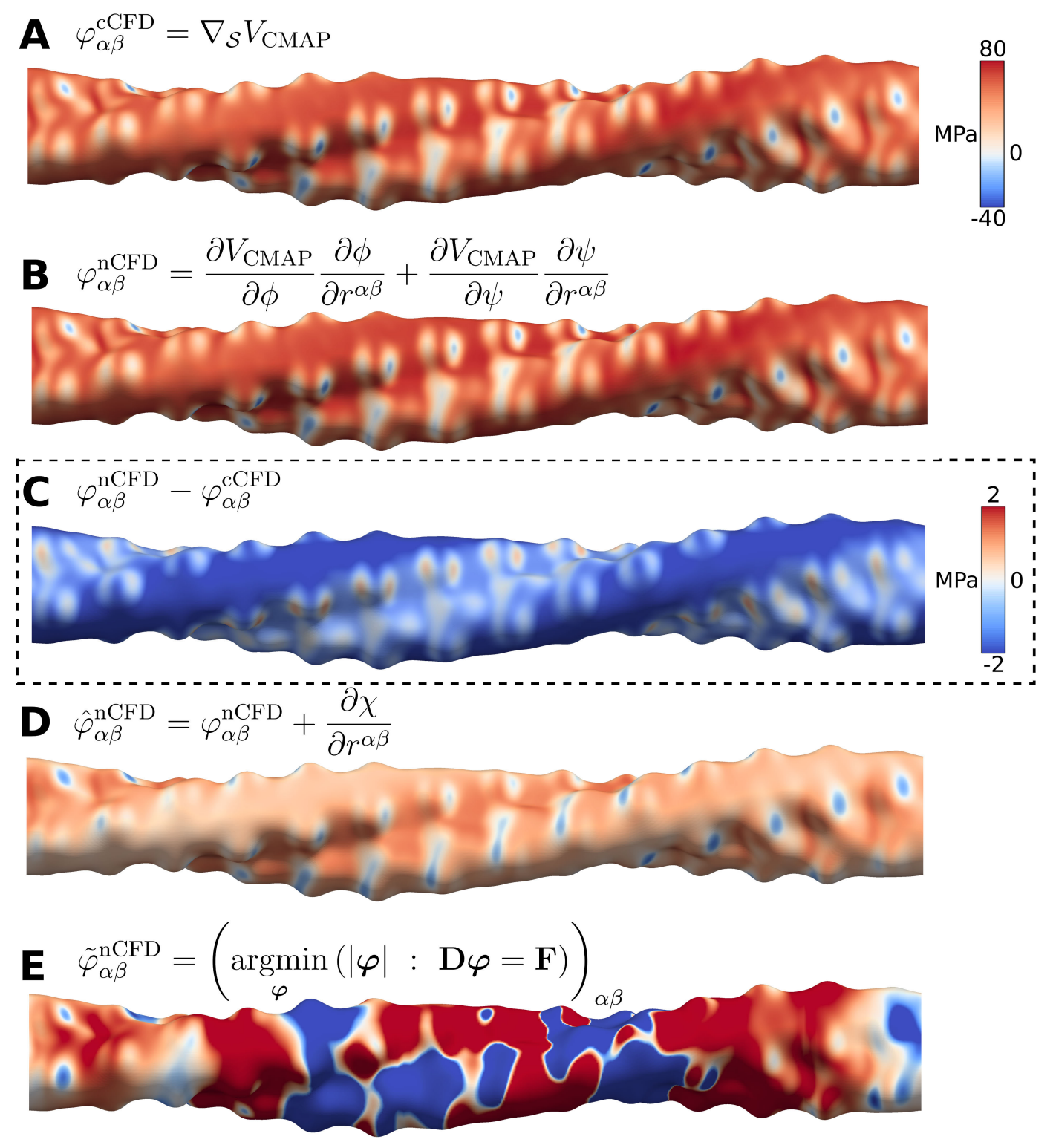

Figure 3: Comparison of the CMAP contribution to the stress from different central force decompositions for a Gaussian filter of $0.2 \mathrm{~nm}$. (A) cCFD, (B) CFD from Eq. (76), (C) Difference between A and B, (D) CFD obtained from the alternative representation $V_{\mathrm{CMAP}}+k \chi$, where $\chi$ is the Caley-Menger determinant from Eq. (66) and $k=10^{6} \mathrm{~nm}^{-5}$, and (E) CFD obtained from Eq. (60) by finding the solution minimizing the norm of $\varphi$. 


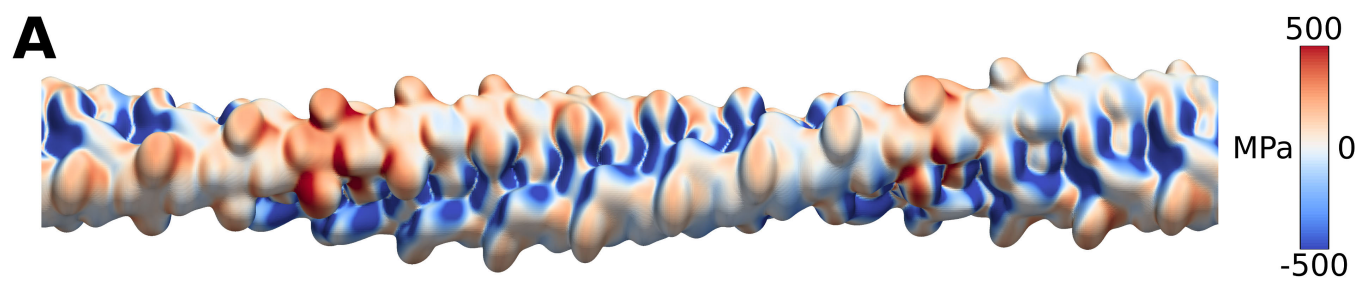

B
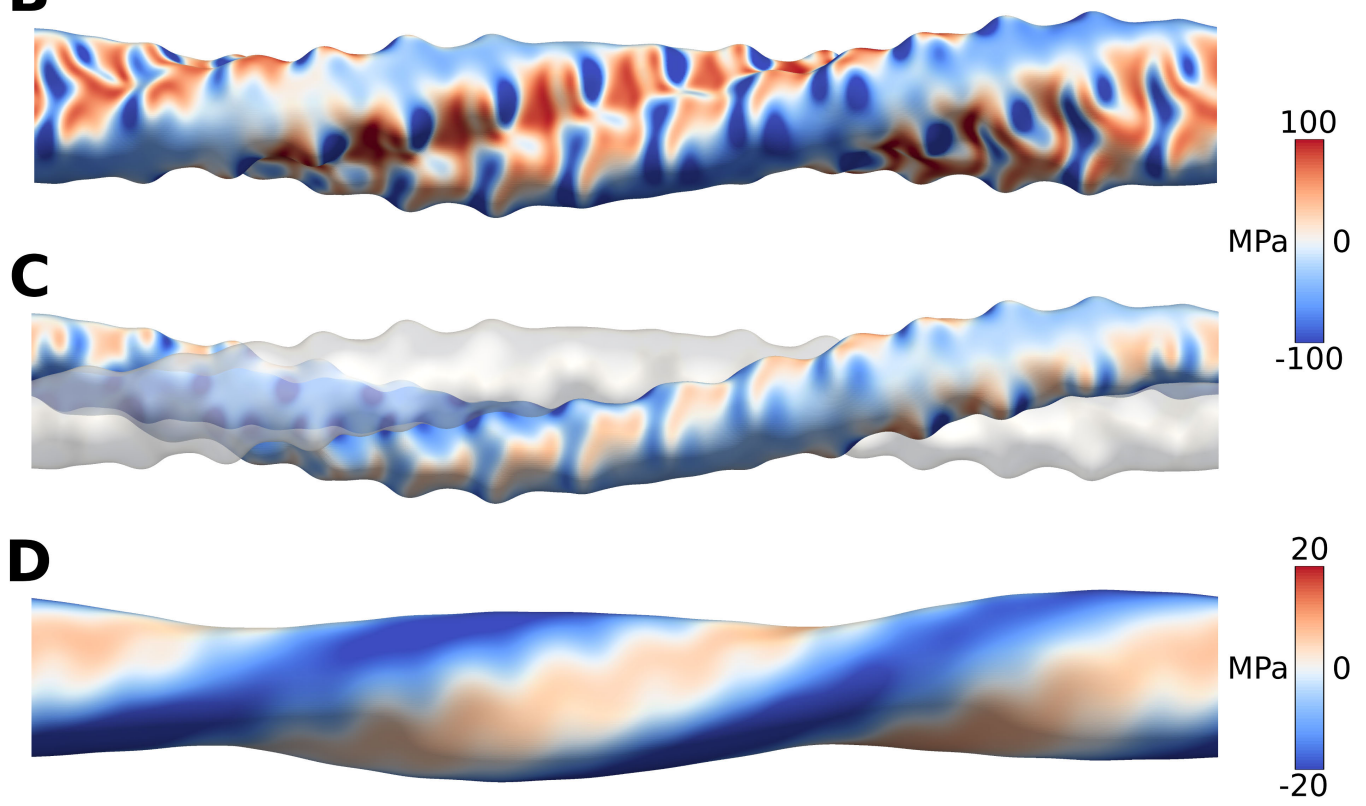

FiguRE 4: Normal traction $t_{n}=\boldsymbol{t} \cdot \boldsymbol{n}$ on the coiled-coil surface for increasing smoothness from Gaussian filters with standard deviations (A) $0.1 \mathrm{~nm}$ (B,C) 0.2 $\mathrm{nm}(\mathrm{D}) 0.4 \mathrm{~nm}$. In (C) we plot the traction on the surface of a single coil. 
observe highly localized tractions, which correlate with interaction sites and molecular features. In particular, we observe high tractions in the zippered region of the protein. As we broaden the spacial extent of the filter $(0.2$ $\mathrm{nm}$ in $\mathrm{B}$ and $\mathrm{C}, 0.4 \mathrm{~nm}$ in $\mathrm{D})$, we progressively smoothen geometric and stress features, lower the magnitudes of the tractions, and loose atomistic details. In D, we still observe two intercalated helical bands of inward and outward tractions that relate to hydrophobic and hydrophilic regions of the protein. We represent in $\mathrm{C}$ the stress on one of the individual coils, showing the confinement at the coil-coil interface due to the hydrophobic effect. In summary, this example illustrates how the microscopic stress can provide insights about the interplay between chemistry and mechanics, and how it can provide a continuum-like picture of a nanoscale system.

\section{Summary and discussion}

Statistical mechanics provides the bridge between particle (microscopic) and continuum (macroscopic) theories, and thus it is very useful to interpret molecular dynamics trajectories from a mechanical viewpoint. However, the mapping from the statistical mechanics of a discrete particle system to a continuum stress field is not unique and depends, for instance, on the method employed to decompose interatomic forces into pairwise terms. We have provided a geometric derivation of the microscopic stress alternative to the classical Irving-Kirkwood theory but consistent with it. In our approach, the stress tensor is defined through the Doyle-Ericksen formula of continuum mechanics rather than through the continuum statement of balance of linear momentum. This procedure naturally selects a canonical force decomposition, irrespective of the many-body nature of the potential, and thus 
generalizes the central force decomposition (CFD) proposed by Admal and Tadmor (2010). Our independent derivation thus supports the physical significance of the CFD. Interestingly, our approach does not rely as the IKN method on Noll's lemma (Noll, 1955). In practice, our method requires computing the covariant derivative of the potential along shape space, which can be efficiently done with algebraic methods. We show here and elsewhere (Torres-Sánchez et al., 2015) that the proposed cCFD results in physically meaningful stress fields in complex protein systems modeled with potentials involving up to 5-body interactions.

One advantage of the usual IKN approach is that it does not require thermodynamic equilibrium, and it is thus more general than our derivation based on the canonical free energy. However, a crucial observation is that the stress tensor obtained with our approach is a particular choice amongst the different IKN stresses resulting from different potential extensions. Thus, because the IKN stress can also be considered in equilibrium conditions, its consistency with the Doyle-Ericksen formula of continuum mechanics, which results in our definition, can be seen as a selection principle amongst different IKN stresses. Thus, taken together, the IKN method and our approach provide a compelling definition of the microscropic stress.

A key aspect in our definition of the microscopic stress is the fact that we consider local shape spaces $\mathcal{S}_{I_{n}}$ suggested by the multibody expansion of the potential. Beyond a practical motivation, this choice can be physically supported because it allows us to localize as much as possible the potential interactions, as discussed in Section 3.6. The cluster expansion is general, irrespective of the way the force-field is defined. Its evaluation, however, 
can become computationally intensive, particularly to fully quantum models. Thus, it remains to be seen if our approach is computationally feasible for such systems.

Our procedure to compute the local stress from the cCFD, along with previous local stress definitions, has been made publicly available in our LIBMDSTRESS library (Vanegas et al.), which can be either used as a standalone library or embedded in the GROMACS 4.5.5 package (Hess et al., 2008).

\section{Acknowledgements}

We acknowledge the support of the European Research Council ERC Grant Agreement nr 240487. ATS acknowledges the support of the Spanish government through Grant No. BES-2012- 054895.

\section{Bibliography}

Nikhil Chandra Admal. Results on the interaction between atomistic and continuum models. PhD thesis, The University of Minnesota, 2014.

Nikhil Chandra Admal and E. B. Tadmor. A unified interpretation of stress in molecular systems. J. Elast., 100(1-2):63-143, May 2010.

Nikhil Chandra Admal and E. B. Tadmor. Stress and heat flux for arbitrary multibody potentials: A unified framework. J. Chem. Phys., 134(18):-, 2011.

Irene Arias and Marino Arroyo. Size-dependent nonlinear elastic scaling of multiwalled carbon nanotubes. Phys. Rev. Lett., 100:085503, Feb 2008. 
Marc Baus and Ronald Lovett. Generalization of the stress tensor to nonuniform fluids and solids and its relation to Saint-Venant's strain compatibility conditions. Phys. Rev. Lett., 65(14):1781-1783, June 1990.

Marc Baus and Ronald Lovett. Stress-strain relations in nonuniform equilibrium fluids. Phys. Rev. A, 44:1211-1218, Jul 1991.

M. Berger and D. Ebin. Some decompositions of the space of symmetric tensors on a riemannian manifold. J. Differential Geom., 3(3-4):379-392, 1969.

Bogdan I Costescu and Frauke Gräter. Time-resolved force distribution analysis. BMC biophysics, 6:5, 2013.

Murray S. Daw and M. I. Baskes. Embedded-atom method: Derivation and application to impurities, surfaces, and other defects in metals. Phys. Rev. B, 29:6443-6453, Jun 1984.

M. P. Do Carmo. Riemannian Geometry. Birkhäuser, Boston, 1992.

T.C. Doyle and J.L. Ericksen. Nonlinear Elasticity. In H.L. Dryden and Th. von Kármán, editor, Advances in Applied Mechanics IV, volume 4, pages 53-115. Elsevier, 1956.

Scott A. Edwards, Johannes Wagner, and Frauke Gräter. Dynamic prestress in a globular protein. PLoS Comput Biol, 8(5):e1002509, 052012.

MichaelE. Fisher. The free energy of a macroscopic system. Arch. Ration. Mech. An., 17(5):377-410, 1964. 
Rüdiger Goetz and Reinhard Lipowsky. Computer simulations of bilayer membranes: Self-assembly and interfacial tension. J. Chem. Phys., 108 (17):7397-7409, 1998.

Harold W Hatch and Pablo G Debenedetti. Molecular modeling of mechanical stresses on proteins in glassy matrices : Formalism. J. Chem. Phys., 137:035103, 2012.

Berk Hess, Carsten Kutzner, David van Der Spoel, and Erik Lindahl. GROMACS 4: Algorithms for highly efficient, load-balanced, and scalable molecular simulation. J. Chem. Theory Comput., 4(3):435-447, March 2008.

Mingyang Hu, Djurre H. de Jong, Siewert J. Marrink, and Markus Deserno. Gaussian curvature elasticity determined from global shape transformations and local stress distributions: a comparative study using the martini model. Faraday Discuss., 161:365-382, 2013.

J. H. Irving and John G. Kirkwood. The statistical mechanical theory of transport processes. IV. The equations of hydrodynamics. J. Chem. Phys., 18(6):817-829, 1950.

Ju Li, Krystyn J Van Vliet, Ting Zhu, Sidney Yip, and Subra Suresh. Atomistic mechanisms governing elastic limit and incipient plasticity in crystals. Nature, 418(6895):307-310, July 2002.

Erik Lindahl and Olle Edholm. Spatial and energetic-entropic decomposition of surface tension in lipid bilayers from molecular dynamics simulations. J. Chem. Phys., 113(9):3882-3893, 2000. 
Robert G. Littlejohn and Matthias Reinsch. Gauge fields in the separation of rotations andinternal motions in the n-body problem. Rev. Mod. Phys., 69:213-276, Jan 1997.

Alexander D MacKerell, Michael Feig, and Charles L Brooks. Improved treatment of the protein backbone in empirical force fields. J. Am. Chem. Soc., 126(3):698-699, January 2004.

Jerrold Marsden and Thomas J. R. Hughes. Mathematical foundations of elasticity. Dover Publications, Inc., New York, New York, USA, 1983.

J W Martin. Many-body forces in metals and the brugger elastic constants. J. Phys. C Solid State, 8(18):2837, 1975.

L Mistura. The definition of the pressure tensor in the statistical mechanics of nonuniform classical fluids. Int. J. Thermophys., 8(3):397-403, 1987.

A. I. Murdoch. Physical foundations of continuum mechanics. Cambridge University Press, 1st edition, 2012.

W Noll. Die herleitung der grundgleichungen der thermomechanik der kontinua aus der statistischen mechanik. J. Rational Mech. and Anal., 4: 627-646, 1955.

O H Samuli Ollila, H J Risselada, Martti Louhivuori, Erik Lindahl, Ilpo Vattulainen, and Siewert J Marrink. 3D pressure field in lipid membranes and membrane-protein complexes. Phys. Rev. Lett., 102:078101, 2009.

Chun-Wei Pao, Stephen M Foiles, Edmund B Webb, David J Srolovitz, and Jerrold A Floro. Atomistic simulations of stress and microstructure evolu- 
tion during polycrystalline Ni film growth. Phys. Rev. B, 79(22):224113, June 2009.

J.M. Porta, L Ros, F Thomas, and C Torras. A branch-and-prune solver for distance constraints. IEEE Trans. Rob, 21(2):176-187, 2005.

G C Rossi and M Testa. The stress tensor in thermodynamics and statistical mechanics. J. Chem. Phys., 132(7):074902, February 2010.

P Schofield and J.R. Henderson. Statistical mechanics of inhomogeneous fluids. Proc. R. Soc. Lond. A, pages 231-246, 1982.

Zhigong Song, Vasilii I. Artyukhov, Boris I. Yakobson, and Zhiping Xu. Pseudo hall-petch strength reduction in polycrystalline graphene. Nano Letters, 13(4):1829-1833, 2013.

Margarita Staykova, Marino Arroyo, Mohammad Rahimi, and Howard A. Stone. Confined bilayers passively regulate shape and stress. Phys. Rev. Lett., 110:028101, 2013.

Ellad B. Tadmor and Ronald E. Miller. Modeling Materials: Continuum, Atomistic and Multiscale Techniques. Cambridge University Press, 2011.

Alejandro Torres-Sánchez, Juan M. Vanegas, and Marino Arroyo. Examining the mechanical equilibrium of microscopic stresses in molecular simulations. Phys. Rev. Lett., 114:258102, Jun 2015.

Juan M Vanegas and M Arroyo. Force transduction and lipid binding in MscL: A continuum-molecular approach. PLoS ONE, 9(12):e113947, 2014. 
Juan M Vanegas, A Torres-Sánchez, and M Arroyo. http://www.lacan.upc.edu/LocalStressFromMD.

Juan M. Vanegas, Alejandro Torres-Sánchez, and Marino Arroyo. Importance of force decomposition for local stress calculations in biomembrane molecular simulations. J. Chem. Theory Comput., 10:691-702, 2014.

Yujie Wei, Jiangtao Wu, Hanqing Yin, Xinghua Shi, Ronggui Yang, and Mildred Dresselhaus. The nature of strength enhancement and weakening by pentagon-heptagon defects in graphene. Nat Mater, 11(9):759-763, 09 2012 .

B. I. Yakobson, C. J. Brabec, and J. Bernholc. Nanomechanics of carbon tubes: Instabilities beyond linear response. Phys. Rev. Lett., 76:25112514, Apr 1996.

Arash Yavari and Jerrold E. Marsden. Energy balance invariance for interacting particle systems. Zeitschrift für angewandte Mathematik und Physik, 60:723-738, 2009.

Arash Yavari and Jerrold E. Marsden. Covariantization of nonlinear elasticity. Zeitschrift für angewandte Mathematik und Physik, 63(5):921-927, 2012. ISSN 0044-2275.

Arash Yavari, Jerrold E. Marsden, and Michael Ortiz. On spatial and material covariant balance laws in elasticity. Journal of Mathematical Physics, 47(4):042903, 2006. 